\title{
Nitric oxide synthase inhibition and oxidative stress in cardiovascular diseases: Possible therapeutic targets?
}

Luc Rochette* ${ }^{*}$, Julie Lorin ${ }^{1}$, Marianne Zeller ${ }^{1}$, Jean-Claude Guilland ${ }^{1}$, Luc Lorgis ${ }^{1,2}$, Yves Cottin $^{1,2}$ and Catherine Vergely ${ }^{1}$

\author{
${ }^{1}$ Laboratoire de Physiopathologie et Pharmacologies Cardio-Métaboliques (LPPCM) \\ ${ }^{2}$ Service de Cardiologie-CHU-Dijon \\ INSERM UMR866 - Université de Bourgogne - Facultés de Médecine et de Pharmacie \\ 7 Boulevard Jeanne d'Arc - 21033 Dijon Cedex - France
}

* Corresponding author: E-mail: luc.rochette@u-bourgogne.fr

\section{ABSTRACT}

Nitric oxide ( ${ }^{\circ} \mathrm{NO}$ ) is synthetized enzymatically from L-arginine (L-Arg) by three NO synthase isoforms, iNOS, eNOS and nNOS. The synthesis of NO is selectively inhibited by guanidino-substituted analogs of L-Arg or methylarginines such as asymmetric dimethylarginine (ADMA), which results from protein degradation in cells. Many disease states, including cardiovascular diseases and diabetes, are associated with increased plasma levels of ADMA. The N-terminal catalytic domain of these NOS isoforms binds the heme prosthetic group as well as the redox cofactor, tetrahydrobiopterin $\left(\mathrm{BH}_{4}\right)$ associated with a regulatory protein, calmodulin (CaM). The enzymatic activity of NOS depends on substrate and cofactor availability. The importance of $\mathrm{BH}_{4}$ as a critical regulator of eNOS function suggests that $\mathrm{BH}_{4}$ may be a rational therapeutic target in vascular disease states. $\mathrm{BH}_{4}$ oxidation appears to be a major contributor to vascular dysfunction associated with hypertension, ischemia/reperfusion injury, diabetes and other cardiovascular diseases as it leads to the increased formation of oxygen-derived radicals due to NOS uncoupling rather than NO. Accordingly, abnormalities in vascular NO production and transport result in endothelial dysfunction leading to various cardiovascular disorders. However, some disorders including a wide range of functions in the neuronal, immune and cardiovascular system were associated with the over-production of NO. Inhibition of the enzyme should be a useful approach to treat these pathologies. Therefore, it appears that both a lack and excess of NO production in diseases can have various important pathological implications. In this context, NOS modulators (exogenous and endogenous) and their therapeutic effects are discussed.

Keywords: free radicals, $\mathrm{NO}$ synthases, $\mathrm{NO}$ inhibitors, $\mathrm{ADMA}, \mathrm{BH}_{4}$, cardiovascular disease 


\section{$\underline{\text { Abbreviations }}$}

ADMA, asymmetric dimethylarginine; AF, atrial fibrillation; AGXT2, alanine-glyoxylate aminotransferase 2; AP-1, activator protein 1; ApoE-KO, apolipoprotein E knockout; ARE, antioxidant response element; $\mathrm{AscH}^{-}$, ascorbate anion; $\mathrm{AT}$, angiotensin; $\mathrm{BH}_{3}$, trihydrobiopterin radical; $\mathrm{BH}_{4}$, tetrahydrobiopterin; CAAs, cationic amino acids; CaM, calmodulin; CAT, catalases; CATs, cationic amino acid transporters; $C R$, carbonyl reductase; CV, cardiovascular; DDAHs, dimethylarginine dimethylaminohydrolases; DHFR, dihydrofolate reductase; DHPR, dihydropteridine reductase; DM, diabetes mellitus; DMA, dimethylamines; DMGV, $\alpha$-keto- $\delta-(N(G), N(G)$-dimethylguanidino)valeric acid; DNTP, 7,8-dihydroneopterin 30 triphosphate; eGFR, estimated glomerular filtration rate; ERK, extracellular signal-regulated kinase; FAD, flavin adenine dinucleotide; FMN, flavin mononucleotide; FOS, Finkel-Biskis-Jinkins osteosarcoma; FOXO, forkhead protein; GPx, glutathione peroxidases; GSH, glutathione; HIF, hypoxia-inducible factor; HSP, heat shock protein; GP, glutathioneperoxidase; GTP, guanosine triphosphate; GTP-CH, GTP cyclohydrolase 1; JNK, c-Jun N-terminal kinase; L-Arg; Larginine; LDL, low-density lipoprotein; L-NMMA, NG -monomethyl-L-arginine; L-NNA), N-nitro-Larginine; LTL, leukocyte telomere length; MAO, monoamine oxidase; MAPK, mitogen-activated protein kinase; MDA, malondialdehyde; MT, metallothionein; NADPH, nicotinamide dinucleotide phosphate NF- $\kappa B$, nuclear factor $\kappa B$; NO, nitric oxide; NOHA, N-omega-hydroxy-L-arginine; NOX, NADPH oxidase; Nrf2, nuclear factor erythroid 2-related factor 2; NTPH, neuronal tryptophan hydroxylase; OGG1, 8-Oxoguanine DNA glycosylase; OS, oxidative stress; PAH, phenylalanine hydroxylase; PCD, pterin-4a-carbinolamine dehydratase; PRMT, protein arginine methyl transferase; Prx, peroxyredoxin; PTPS, 6-pyruvoyl tetrahydropterin synthase; Ref-1, redox factor-1; ROS, reactive oxygen species; RNS, reactive nitrogen species; SAH, S-adenosyl-L-homocysteine; SAM, S-adenosyl-Lmethionine; SDMA, symmetric dimethylarginine; siRNAs, small interfering ribonucleic acids; SOD, superoxide dismutase; $S R$, sepiapterin reductase; $T H$, tyrosine hydroxylase; tHcy, total plasma homocysteine; TocH, tocopherol; Trx, thioredoxin; UA, uric acid; $y+L A A, y+L A T$ transporters. 


\section{Contents}

\section{Introduction}

\section{Free radicals and oxidative stress}

\section{Nitric oxide and nitric oxide synthases}

3.1. Production of nitric oxide: a free radical

3.2. Molecular structure of NO synthases

3.3. Regulation and uncoupling of NOS

$$
\begin{aligned}
& \text { Importance of L-arginine metabolism } \\
& \text { Importance of } \mathrm{BH}_{4} \\
& \text { Uncoupling of NOS }
\end{aligned}
$$

\section{Endogenous NO synthase inhibitors}

4.1. Proteolysis and formation of ADMA by PRMTs

4.2. Metabolism of ADMA by DDAHs

4.3. Metabolism of ADMA and oxidative stress

\section{Cardiovascular and metabolic consequences of increased ADMA concentrations}

5.1. Endothelial dysfunction and ADMA

5.2. ADMA and cardiovascular diseases

ADMA and cardiovascular risk factors

ADMA, heart failure and hypertension

5.3. ADMA and mortality

5.4. Metabolic parameters and ADMA

Diabetes

Metabolic syndrome and Insulin resistance

Atherosclerosis 


\section{NO synthases/ADMA - Therapeutic targets}

6.1. Substrate availability: L-Arginine

6.2. eNOS transcription enhancers

6.3. Treatment with $\mathrm{BH}_{4}$

6.4. Treatment with folic acid

6.5. Treatment with trans-resveratrol

6.6. Treatments with statins

6.7. Drugs that interfere with the renin-angiotensin-aldosterone system

6.8. Modulation of DDAH or PRMT activity as a therapeutic target

DDAH activity

PRMT activity

6.9. Selective NADPH oxidase (NOX) inhibitors

Natural NADPH oxidase inhibitors

Chemical NADPH oxidase inhibitors

\section{Development of nitric oxide synthases inhibitors as therapeutic agents}

\section{Summary and future directions}

\section{References}




\section{Introduction}

The discovery of the biological ambivalence of oxidative stress, as well as its numerous metabolic, structural, and functional effects, has generated a large number of experimental and clinical investigations concerning the association between nitric oxide free radical ( $\mathrm{NO}$ ) metabolism and disease. NO is synthetized enzymatically from L-Arg by NO synthases. Very intriguing, it has been observed that eNOS itself can be a source of superoxide, and thereby cause endothelial dysfunction. It appears that eNOS may become "uncoupled". The synthesis of NO is selectively inhibited by guanidino-substituted analogs of L-Arg or methylarginines such as asymmetric dimethylarginine (ADMA), which results from protein degradation in cells. ADMA accumulates in various disease states and its concentration in plasma is strongly predictive of cardiovascular diseases. Given the clinical properties of endothelial NO, eNOS and ADMA are interesting targets for the prevention or treatment of cardiovascular diseases. Oxidative stress is probably the main cause of oxidation of the essential NOS cofactor, tetrahydrobiopterin $\left(\mathrm{BH}_{4}\right)$. A lack of $\mathrm{BH}_{4}$ leads to eNOS uncoupling (i.e., uncoupling of oxygen reduction from NO synthesis in eNOS). Abnormalities in vascular NO production and transport result in endothelial dysfunction resulting in various cardiovascular pathologies like hypertension, atherosclerosis and angiogenesis-associated disorders. However, some disorders, including those that affect a wide range of functions in the neuronal, immune and cardiovascular system (inflammation, septic shock,..) were associated with the over-production of NO. Inhibition of the enzyme should be a useful approach to treat these pathologies. Therefore, it appears that both a lack and excess of NO production in diseases can have various important pathological implications. In this context, NOS modulators (exogenous and endogenous) and their therapeutic effects are discussed in this review.

\section{Free radicals and oxidative stress}

Free radicals, known in chemistry since the beginning of the $20^{\text {th }}$ century, were initially used to describe intermediate compounds in organic and inorganic chemistry, and several chemical definitions for them were suggested. Free radicals can be defined as molecules or molecular fragments containing one or more unpaired electron in molecular orbitals. These unpaired electrons make free radicals extremely reactive. Reactive oxygen species (ROS) are produced as intermediates in reduction-oxidation (redox) reactions. Radicals derived from oxygen (ROS) and nitrogen (RNS: derived from NO) constitute the most important class of radical species generated in living systems. ROS are formed in both reducing and oxidizing conditions on the electron acceptor and donor side of PSII, respectively: superoxide anion radical $\left(\mathrm{O}_{2}{ }^{-}\right)$hydrogen peroxide $\left(\mathrm{H}_{2} \mathrm{O}_{2}\right)$ and hydroxyl radical $\left({ }^{\circ} \mathrm{OH}\right)$. ROS and RNS are products of normal cellular metabolism and are well recognized for their dual role as both deleterious and beneficial species (Halliwell \& Gutteridge, 1995; C. Vergely, Maupoil, Clermont, Bril, \& Rochette, 2003). The most important sources of the ROS generated in endothelial cells include nicotinamide dinucleotide phosphate (NADPH) oxidases (NOX), xanthine oxidase, mitochondria, and, under specific circumstances, endothelial NO synthases (Montezano \& Touyz, 2012). Under certain conditions (e.g. upon stimulation with angiotensin II), the activity of NOX is increased in endothelial and smooth muscle cells, suggesting that in the presence of an activated renin-angiotensin system (either local or circulating), vascular dysfunction due to increased vascular 
superoxide production is likely (Oudot, et al., 2006; Richard, et al., 2008). Xanthine oxidase catalyzes the sequential hydroxylation of hypoxanthine to yield xanthine and uric acid (UA). The enzyme can exist in two forms that differ primarily in the specificity of their oxidizing substrates. Increased UA levels may be an indicator of up-regulated activity of xanthine oxidase, a powerful oxygen radicalgenerating system in human physiology.

Some oxygen-derived radicals with a short half-life are extremely reactive. For example, ${ }^{\circ} \mathrm{OH}$ can survive for $10^{-10} \mathrm{sec}$ in biological systems. The lifespan of other radicals is also short but depends on their environment. For example, superoxide ion radical possesses different properties depending on the environment and $\mathrm{pH}$. Given its $\mathrm{pKa}$ of 4.8 , superoxide can exist in the form of either $\mathrm{O}_{2}{ }^{-}$or, at a low $\mathrm{pH}$, as hydroperoxyl: $\mathrm{HO}_{2}{ }^{\circ}$. The latter can more easily penetrate biological membranes than the charged form.

Mitochondria are the major source of ROS production within the cell. Increased levels of ROS are a likely culprit in a variety of pathophysiological conditions, including cardiovascular diseases and diabetes. Mitochondria have long been known to generate significant quantities of $\mathrm{H}_{2} \mathrm{O}_{2}$. The hydrogen peroxide molecule does not contain an unpaired electron and is thus not a radical species. In physiological conditions, the production of hydrogen peroxide is estimated to account for about $\sim 2 \%$ of the total oxygen uptake by the organism.

In the context of ROS and RNS, the concept of "oxidative stress" (OS) is frequently used in a number of biochemical, physiological and pathophysiological situations. It describes the result of an increase in the production and/or a decrease in the elimination of reactive species. OS is also often defined as an imbalance of pro-oxidants and antioxidants. The organism must confront and control the presence of both pro-oxidants and antioxidants continuously. Under normal conditions, there is a balance between both the activities and the intracellular levels of these antioxidants.

Increased OS within mitochondria arising from impaired oxidative metabolism may contribute to greater lipid peroxidation and damage to cell membranes and DNA, activating a cascade of signaling events that further exacerbate the severity of the disease. A number of studies have found that altering mitochondrial fusion and fission events can influence ROS production in mitochondria (Gruber, Schaffer, \& Halliwell, 2008). In addition to the electron transport system, mitochondria also generate $\mathrm{H}_{2} \mathrm{O}_{2}$ from monoamine oxidase (MAO) bound to the outer membrane. MAO is the enzyme responsible for the metabolism of catecholamine, and has recently been shown to be a substantial source of $\mathrm{H}_{2} \mathrm{O}_{2}$ and oxidative stress in the mesenteric arteries of patients with type 2 diabetes (Nunes, et al., 2011).

Exposure to free radicals from a variety of sources has led organisms to develop defense mechanisms via antioxidant agents. Several classes of antioxidant agents may be considered, but it is important to clarify some points concerning the specificity of each antioxidant agent. An antioxidant can be defined as any substance that, when present in very low concentrations compared to that of an oxidizable substrate, significantly delays or inhibits the oxidation of that substrate. Defense mechanisms against free radical-induced OS involve: (1) preventive mechanisms, (2) repair mechanisms, (3) antioxidant defenses. Enzymatic antioxidant defenses include superoxide dismutases (SOD), glutathione peroxidases (GPX), catalases (CAT) and other enzymes such as 
peroxiredoxin or thioredoxin (Trx). Non-enzymatic antioxidants include vitamins, ascorbic acid (Vitamin C), tocopherol (Vitamin E), and other direct antioxidants such as glutathione (GSH), folic acid, lipoic acid, thiols and indirect antioxidants (chelate redox metals, pharmacological drugs). To prevent the interaction between radicals and biological targets, the antioxidant should be present at the location where the radicals are being produced in order to compete with the radical for the biological substrate. Because most radicals are short-lived species, they react quickly with other molecules. In the body, metal ion excess or deficiency can potentially inhibit protein function, interfere with correct protein folding or, in the case of iron or copper, promote oxidative stress (Cottin, et al., 1998; Puntarulo, 2005; Rochette, et al., 2011). The involvement of metal ions in disorders has made them an emerging target for therapeutic interventions. Iron, an essential element for many important cellular functions in all living organisms, can catalyze the formation of potentially toxic free radicals. Excessive iron is sequestered by ferritin in a nontoxic and readily available form in a cell (Uriu-Adams \& Keen, 2005).

Recently, the theory of oxidative stress has been extended to account for an alternative mechanism: a disruption of thiol-redox circuits, which leads to aberrant cell signaling and dysfunctional redox control (Jones, 2006). Research tools are becoming available to elucidate details of subcellular redox organization, and this development highlights an opportunity for a new generation of targeted antioxidants to enhance and restore redox signaling (Jones \& Go, 2010; Roede, Go, \& Jones, 2012). It has been proposed that the aging process itself is regulated by this oxidative stress imbalance (Martin, et al., 2007; Oudot, et al., 2006).

Among the many methods that can be used to measure oxidant and free-radical generation, electron paramagnetic resonance (EPR) spectroscopy is one of the few which enables the reliable, direct detection of free radicals. In the past, we successfully used EPR spin-trapping or spin-probing in biological systems in order to identify and quantify reactive species in conditions associated with cardiovascular diseases (Oudot, et al., 2006; C Vergely, Maupoil, Benderitter, \& Rochette, 1998; C. Vergely, et al., 2003; Catherine Vergely, Tabard, Maupoil, \& Rochette, 2001). However, this technique requires complex preparation of the samples, and is difficult to apply in clinical practice due to technical constraints. Ways to measure OS have been developed: e.g. determination of the total plasma antioxidant status or the plasma malondialdehyde (MDA) concentration (Ben Baouali, Aube, Maupoil, Blettery, \& Rochette, 1994). Recently, a new test has been developed: the free oxygen radicals test (FORT), which was designed as a point-of-care system using freshly collected heparinized whole blood. Strong correlations between the FORT index and other indices of inflammation or oxidative stress have been reported. The FORT technique is faster and particularly suitable for bedside conditions. This simple tool, which allows the measurement of circulating ROS, may thus encourage the study of relationships between ROS concentrations and markers of cardiovascular diseases. The FORT can be used to assess circulating oxidative stress in patients with acute myocardial infarction (Lorgis, et al., 2010).

\section{Nitric oxide and nitric oxide synthases}


-NO has a half-life of only a few seconds in an aqueous environment after being synthetized from LArg by NO synthases (NOS). Three distinct isoforms of NOS have been identified, all of which have different characteristics and generate NO at different rates. NO is a free radical and can stabilize its unpaired electron by two mechanisms: reaction with species containing other unpaired electrons (thus pairing up the two lone electrons) and interaction with the $d$-orbitals of transition metals, particularly iron. NO functions as an intracellular messenger stimulating guanylate cyclase, and thereby relaxing smooth muscles in blood vessels. It is not a locally-acting messenger and the spatial distribution of NO in tissue is large. NO is uncharged and highly soluble in hydrophobic environments. This property may allow it to diffuse freely across biological membranes and to signal many cell diameters from its site of generation (Michel \& Vanhoutte, 2010). NO is an important signaling molecule for the regulation of vital functions. "NO reacts with molecular oxygen and ROS to generate a range of oxidation products such as the different RNS. One well-characterized RNS-forming reaction is that of ${ }^{\circ} \mathrm{NO}$ with superoxide $\left(\mathrm{O}_{2}{ }^{\circ}\right)$ which occurs at nearly diffusion-limited rates to produce peroxynitrite: $\mathrm{ONOO}^{-}$. It is itself strongly oxidizing, and when protonated undergoes homolytic scission to produce hydroxyl radical $\left({ }^{\circ} \mathrm{OH}\right)$ and nitrogen dioxide $\left({ }^{\circ} \mathrm{NO}_{2}\right)$. Recently, the term "oxygenomics" has been proposed for the research area involved in the molecular changes of nucleic acids that occur in various pathological states induced by oxidative stress (Toyokuni, 2008). Additional reactive radicals derived from a number of endogenous molecules can be formed in living systems. These include carbon monoxide, nitrogen dioxide and hydroperoxyl. The last decade had seen growing interest in the biology of endogenous, biologically active gases such as NO, CO and hydrogen disulfide $\left(\mathrm{H}_{2} \mathrm{~S}\right)$. All three gases are thought to play several roles in inflammation associated with diabetes (Rochette, Cottin, Zeller, \& Vergely, 2013; Rochette \& Vergely, 2008).

A number of techniques are used to detect NO in cell suspensions and isolated tissue preparations. In general, electron paramagnetic resonance (EPR) spectroscopy is considered the best technique available for the detection and measurement of radical production. EPR methods require the stabilization of NO using endogenous and exogenous spin traps. Spin trapping in combination with EPR can characterize NO in real time and at their site of production. The most frequently used iron chelates for the in vivo spin trapping of NO are iron-N-methyl D-glucamine dithiocarbamate $\left(\mathrm{Fe}(\mathrm{MGD})_{2}\right)$ and iron-diethyl dithiocarbamate (Fe-DETC). MGD is able to diffuse into the tissue and to selectively bind NO in biological systems after complexing with reduced iron Fe(II). The NO-FeMGD complex formed gives rise to a characteristic triplet EPR spectrum and is a relatively stable product (Clermont, et al., 2003). The process of NO transfer into erythrocytes is of critical biological importance because it controls plasma NO bioavailability and the diffusion distance of endothelialderived NO (Clermont, et al., 2003; Fink, Dikalov, \& Fink, 2006; Lecour, Maupoil, Siri, Tabard, \& Rochette, 1999; C. Vergely, et al., 2003).

\subsection{Molecular structure of NO synthases (Figure 1)}

In mammals, three main NOS isozymes, neuronal (nNOS or NOS I), inducible (iNOS or NOS II), and endothelial (eNOS or NOS III) NOS catalyze this reaction. NO is produced by iNOS in the immune system. The reaction mechanism of NOS presents many similarities with that of cytochrome P450. NOS is a large enzyme ranging in size from 135 to $160 \mathrm{kDa}$ but the monomeric form is inactive. The formation of a tight dimer through the heme domains is required for activity. NOS catalyze a two- 
step reaction that converts L-Arg through an intermediate, N-omega-hydroxy-L-arginine (NOHA), to the final products, NO and citrulline. Concerning the localization of NOS, eNOS is predominantly targeted to the sarcolemmal caveolae, which are invaginations of the plasma membrane. In the periphery, many smooth muscle tissues are innervated by nitrergic nerves, i.e. nerves that contain nNOS and generate and release NO. NO produced by nNOS in nitrergic nerves can be viewed as an unusual neurotransmitter that stimulates NO-sensitive guanylyl cyclase in its effector cells, thereby decreasing the tone of blood vessels (Daff, 2010).

All three NOS isoforms, eNOS, nNOS, and iNOS, are self-sufficient enzymes with two major functional domains fused into a single polypeptide. The structures of iNOS, eNOS and nNOS have been determined (Daff, 2010; Y. H. Zhang \& Casadei, 2012). The N-terminal catalytic domain binds the heme prosthetic group as well as the redox cofactor, tetrahydrobiopterin $\left(\mathrm{BH}_{4}\right)$ associated with a regulatory protein, calmodulin (CaM). The C-terminal reductase domain has binding sites for flavin mononucleotide (FMN), flavin adenine dinucleotide (FAD), and NADPH. Both nNOS and eNOS are expressed constitutively in mammals and called constitutive NOS (cNOS). cNOS activity is controlled by CaM binding in a $\mathrm{Ca}^{2+}$ concentration-dependent manner. The enzyme is bound to caveolin-1, a protein that inhibits eNOS activity. Caveolin-1 interacts with the CaM binding sites and inhibits electron transfer from NADPH at the reductase domain to the heme molecule in the oxygenase domain (Daff, 2010; Gielis, et al., 2011). NO synthesis is driven by electron transfer through FAD and FMN cofactors, and is controlled by CaM binding in constitutive mammalian enzymes (Roman \& Masters, 2006). Electron transfer from FMN to heme has been shown to be the rate-determining step for overall NO synthesis and involves a large-scale conformational change in which the FMN domain moves from an electron input state to an electron output state in which the FMN is close enough to the heme to transfer an electron (Gielis, et al., 2011).

A complementary approach is to manipulate the genes that encode NOS enzymes to generate knockout mice in which a particular NOS gene has been disrupted (H. Liu, et al., 2008). Targeted disruption of the nNOS, iNOS and eNOS genes in mice has led to the development of mutant mice that have been useful tools with which to study how NO cardiovascular parameters associated with endothelial dysfunction, response to vascular injury, and ischemia-reperfusion or atherosclerosis.

\subsection{Regulation and uncoupling of NOS}

Importance of L-arginine metabolism

The enzymatic activity of NOS is tightly controlled, and depends on substrate and cofactor availability, as well as on the rate of electron transfer. The substrate and cofactors that control NOS activity are also involved in other major metabolic pathways in the cell, thereby connecting NOS activity with other metabolic pathways. In the presence of sufficient cofactors ( $\mathrm{NADPH}, \mathrm{FMN}, \mathrm{BH}_{4}$, FAD), NOS activity is dependent on the availability of L- Argand oxygen (Thomas, et al., 2008).

The regulation of L-Arg transport is essential in the regulation of NO synthesis. Under normal circumstances, only $1.2 \%$ of plasma L-Arg turnover is associated with NO formation. Although small, the plasma arginine compartment contributes significantly $(\sim 60 \%)$ as a precursor pool for the 
synthesis of whole body NO. Intracellular concentrations of L-Arg range from 0.1 to $0.8 \mathrm{mM}$ in cultured endothelial cells while plasma L-Arg levels in humans reportedly range from 80 to $120 \mu \mathrm{M}$ although levels as high as $210 \mu \mathrm{M}$ under normal physiological conditions have been reported (Wu \& Morris, 1998). Despite the substantial intracellular concentration of L-Arg, extracellular L-Arg has been demonstrated to be rate limiting for NO production. Multiple transport systems mediate the influx of cationic and anionic amino acids across plasma membranes into mammalian cells. Cationic amino acid transporters (CATs) ensure cellular L-arginine requirements are met via the uptake of extracellular L-Arg (Chin-Dusting, Willems, \& Kaye, 2007).

\section{Importance of $\mathrm{BH}_{4}$ (Figure 2)}

The importance of 2-amino-6-(1,2-dihydroxypropyl)-5,6,7,8-tetrahydro- $1 \mathrm{H}$-pteridin-4-one $\left(\mathrm{BH}_{4}\right)$ as a critical regulator of eNOS function suggests that $\mathrm{BH}_{4}$ may be a rational therapeutic target in vascular disease states. Loss of $\mathrm{BH}_{4}$ appears to be the main mechanism of NOS uncoupling and logically the most likely therapeutic target. Disorders of biopterin synthesis and regeneration were initially identified in patients with phenylketonuria. Indeed, several studies have already explored the effect of $\mathrm{BH}_{4}$ administration on endothelial functions. Mouse models have become an essential tool in biomedical research for the study of gene function including $\mathrm{BH}_{4}$ deficiency and its pathology. Mouse models are available for most of the primary defects in $\mathrm{BH}_{4}$ metabolism, such as cofactor biosynthesis and regeneration, but also for $\mathrm{BH}_{4}$ cofactor-dependent enzymes (Werner, Blau, \& Thony, 2011).

$\mathrm{BH}_{4}$ is formed de novo from guanosine triphosphate (GTP), via a sequence of enzymatic steps mediated by GTP cyclohydrolase 1 (GTP-CH), 6-pyruvoyl tetrahydropterin synthase (PTPS) and sepiapterin reductase (SR). In the first, and rate-limiting step, GTP is reduced to 7,8-dihydroneopterin 30 triphosphate (DNTP) by GTP-CH (Crabtree, Smith, Lam, Goligorsky, \& Gross, 2008). GTP-CH protein levels have been shown to be induced by $\mathrm{H}_{2} \mathrm{O}_{2}$ in oxidative stress (Shimizu, et al., 2008). $\mathrm{BH}_{4}$ is one of the most potent naturally occurring reducing agents. It is therefore reasonable to hypothesize that oxidative stress may lead to excessive oxidation and depletion of $\mathrm{BH}_{4}$. $\mathrm{BH}_{4}$ was found to stabilize the dimeric form of $\mathrm{NOS}_{\text {. }} \mathrm{BH}_{4}$ provides the two electrons required for the reduction of the second atom to water and therefore acts as substrate rather than a tightly bound cofactor. A crucial step in the elucidation of the reaction mechanism was the detection of a trihydrobiopterin radical $\left(\mathrm{BH}_{3}\right)$. The iron is present as a heme ion. Heme and $\mathrm{BH}_{4}$ are found in the oxygenase domain of the enzyme, the additional cofactors FAD and FMN bind to the reductase domain, which donates electrons from NADPH to the oxygenase domain. NOSs are active as dimers, and the reductase domain of one dimer interacts with the oxygenase domain of the other dimer (Werner, et al., 2011).

$\mathrm{BH}_{4}$ distribution is highly tissue-specific in mammals, suggesting the presence of a tissue-specific regulatory mechanism to maintain cellular $\mathrm{BH}_{4}$ homeostasis. Although $\mathrm{BH}_{4}$ is the predominant form of biopterins in normal plasma or cells, it is prone to oxidation to 7,8-dihydrobiopterin $\left(\mathrm{BH}_{2}\right)$ by intracellular ROS. Inactive $\mathrm{BH}_{2}$ is reduced back to $\mathrm{BH}_{4}$ by regeneration enzymes to replenish cellular $\mathrm{BH}_{4}$ levels. The $\mathrm{BH}_{4} / \mathrm{BH}_{2}$ ratio as well as $\mathrm{BH}_{4}$ levels determines the fate of $\mathrm{NO}$ (H. L. Kim \& Park, 2010). $\mathrm{BH}_{4}$ can be oxidized by $\mathrm{ROS}$, leading to a reduction in vascular $\mathrm{BH}_{4}$, an increase in vascular $\mathrm{BH}_{2}$ availability and a decrease in eNOS function thus inducing an increase in ROS production associated with a decrease in NO production. Recently, it has been reported that dietary co-supplementation 
with $\mathrm{BH}_{4}, \mathrm{~L}-\mathrm{Arg}$, and Vitamin $\mathrm{C}$ acted synergistically to decrease $\mathrm{OS}$, increase $\mathrm{NO}$ and improve blood flow in response to acute hind-limb ischemia. Co-supplementation with $\mathrm{BH}_{4}+\mathrm{L}$-Arg + Vitamin $\mathrm{C}$ resulted in greater eNOS activity and NO concentration as well as greater blood flow recovery in the feet of rats with hind-limb ischemia than was the case in those receiving normal chow or either agent separately (Yan, Tie, \& Messina, 2012). 
In most situations where endothelial dysfunction due to increased OS is encountered, the expression of eNOS has been shown to be paradoxically increased rather than decreased. It appears that eNOS may become "uncoupled". In this uncoupled state, electrons normally flowing from the reductase domain of one subunit to the oxygenase domain of the other subunit are diverted to molecular oxygen rather than to L-Arg; in these conditions, superoxide rather than NO is produced. Superoxide produced from uncoupled NOS leads to OS (Roe \& Ren, 2012). The most prominent cause of NOS uncoupling is the loss of the critical NOS cofactor $\mathrm{BH}_{4}$ either by oxidation, or decreased expression of the recycling enzyme dihydrofolate reductase (DHFR) (L. Zhang, et al., 2007).

As we have already reported in the setting of activation of a vascular superoxide source, superoxide will react with $\mathrm{NO}$ to form the highly reactive intermediate peroxynitrite. Peroxynitrite in turn will oxidize $\mathrm{BH}_{4}$ to the $\mathrm{BH}_{3}{ }^{\cdot}$ radical, and the resulting decrease in endothelial $\mathrm{BH}_{4}$ triggers eNOS uncoupling. Oxidation of $\mathrm{BH}_{4}$ not only reduces $\mathrm{BH}_{4}$ bioavailability, but the oxidation products such as $\mathrm{BH}_{2}$ may compete with $\mathrm{BH}_{4}$ for binding to eNOS, thereby leading to eNOS uncoupling. Interestingly, vitamin $\mathrm{C}$ was able to recycle the $\mathrm{BH}_{3}{ }^{\cdot}$ radical to $\mathrm{BH}_{4}$ but not $\mathrm{BH}_{2}$ to $\mathrm{BH}_{4}$. This observation may indicate that the beneficial effects of vitamin $\mathrm{C}$ on endothelial function in patients may be explained in part by the recycling of the $\mathrm{BH}_{3}{ }^{\circ}$ radical to $\mathrm{BH}_{4}$, rather than by directly scavenging superoxide (Kuzkaya, Weissmann, Harrison, \& Dikalov, 2003). An important mechanism whereby ascorbate stabilizes levels of $\mathrm{BH}_{4}$ seems to involve the reduction of the $\mathrm{BH}_{3}{ }^{\cdot}$ radical back to $\mathrm{BH}_{4}$, rather than the prevention of oxidation of $\mathrm{BH}_{4}$ by oxidants such as $\mathrm{ONOO}^{-}$. In this context, the antioxidant reaction of vitamin $\mathrm{E}$ (tocopherols, TocH's) associated with the production of a vitamin E radical (tocopheroxyl radical, $\mathrm{Toc}^{\circ}$ ) has been demonstrated. $\mathrm{TocH}$ is regenerated by the reaction of Toc with vitamin $\mathrm{C}$ (ascorbate monoanion, $\mathrm{AscH}^{-}$) at the interface of the cell membrane and the water phase. $\mathrm{Toc}^{\circ}+\mathrm{AscH}^{-} \rightarrow \mathrm{TocH}+\mathrm{Asc}^{\cdot-}$ where $\mathrm{Asc}^{\cdot-}$ denotes the dehydroascorbate monoanion radical (Figure 3).

Another demonstration of the concept that $\mathrm{BH}_{4}$-mediated eNOS uncoupling contributes to endothelial dysfunction was provided by experiments demonstrating that supplementation with $\mathrm{BH}_{4}$ or with the $\mathrm{BH}_{4}$ precursor sepiapterin was able to improve endothelial dysfunction (Forstermann \& Sessa, 2012; Wunderlich, et al., 2008). Recently, it has been reported that the protection of soluble guanylate cyclase against oxidative inactivation may contribute to the known beneficial effects of $\mathrm{BH}_{4}$ in cardiovascular disorders associated with oxidative stress (Schmidt, et al., 2012). Drugs that interfere with the renin-angiotensin-aldosterone system and statins (3-hydroxy-3-methylgultarylcoenzyme A reductase inhibitors) are able to prevent endothelial dysfunction and eNOS uncoupling (Forstermann \& Sessa, 2012). It has been postulated a role of eNOS uncoupling for reduced number and function of endothelial progenitor cells (EPC), key regulators of vascular repair (Hagensen, Vanhoutte, \& Bentzon, 2012). In diabetes mellitus, EPC levels in diabetic patients were significantly reduced as compared with those of control subjects and showed impaired migration capacity (Thum, et al., 2007). Subsequent reduction of EPC levels and functions likely contributes to the pathogenesis of vascular disease in diabetes (Fadini, et al., 2012; Hamed, Brenner, \& Roguin, 2011; Kuliszewski, et al., 2012).

In conclusion, a major contributor to vascular dysfunction associated with hypertension, ischemia/reperfusion injury, diabetes and other cardiovascular diseases appears to be an effect of 
oxidized $\mathrm{BH}_{4}$, which leads to the increased formation of oxygen-derived radicals, due to NOS uncoupling, rather than NO. Optimal concentrations of $\mathrm{BH}_{4}$ are important for the normal function of eNOS in endothelial cells. In these conditions, $\mathrm{BH}_{4}$ appears to be a rational therapeutic target in a number of vascular diseases.

\section{Endogenous NO synthases inhibitors}

\subsection{Proteolysis and formation of ADMA by PRMTs (Figure 4)}

Asymmetric methylarginines inhibit NO synthesis in vivo by competing with L-Arg at the active site of NO synthases. The synthesis of NO is selectively inhibited by guanidino-substituted analogs of L-Arg, such as $\mathrm{N}^{G}$-monomethyl-L-arginine (L-NMMA) or N-nitro-L-arginine (L-NNA), which act as competitive inhibitors at the active site of the enzyme (Rees, Palmer, Schulz, Hodson, \& Moncada, 1990). Vallance et al. (Vallance, Leone, Calver, Collier, \& Moncada, 1992) reported that L-NMMA and asymmetric dimethylarginine (ADMA) act as endogenous inhibitors of NO synthase. ADMA belongs to a group of naturally occurring methylarginines, which are by-products of protein degradation in cells (proteolysis of post-translationally methylated tissue proteins). ADMA is continuously formed during the intracellular turnover of proteins. Methylated arginine residues are released into the cytoplasm from the breakdown of proteins that have been acted on by enzymes: protein-arginine methyl transferases (PRMT) 1 and 2; methylation being an important mechanism in biology (Bedford \& Clarke, 2009). In mammalian cells, these enzymes have been classified into type I (PRMT1, 3, 4, 6, and 8) and type II (PRMT5, 7, and FBXO11) enzymes, depending on their specific catalytic activity. Both types of PRMT, however, catalyze the formation of monomethylarginine (MMA) from L-Arg. In the second step, type I PRMTs produce asymmetric dimethylarginine (ADMA), while type II PRMT catalyzes symmetric dimethylarginine (SDMA). Proteins containing methylarginine groups lead to the release of free methylarginine into the cytoplasm. Free methylarginines are cleared from the plasma by renal excretion and hepatic metabolism. MMA and ADMA can be degraded to citrulline and dimethylamines (DMA) by dimethylarginine dimethylaminohydrolases (DDAHs) (Pope, Karuppiah, \& Cardounel, 2009). ADMA can be exported from the cell to the circulation via cationic amino acid transporters (CAT), which also mediate uptake by other cells or organs, thereby facilitating interorgan transport of ADMA (Davids, et al., 2012). Whole blood contains large amounts of proteinincorporated ADMA. The role of erythrocytes in the storage and generation of the endogenous NOS inhibitor ADMA has been investigated. There is fast bidirectional traffic of ADMA across the plasma membrane of the erythrocyte, leading to equilibrium between intra- and extracellular ADMA. Upon lysis of erythrocytes, proteolytic activity leads to a substantial release of free ADMA from methylated proteins but with no appreciable contribution from hemoglobin. The erythrocyte serves as both a reservoir and a source of free ADMA (Davids, et al., 2012). ADMA is both exported from its site of origin, and imported from the plasma at distant sites, by CATs in exchange for arginine and other cationic amino acids (CAAs). The critical step that determines the distribution of ADMA between the cytosol and the extracellular fluid is its transmembrane transport via CATs. CATs are widely distributed on cell membranes either as high-affinity, low-capacity transporters, exemplified by CAT1 , which transport ADMA and arginine across cell membranes in blood vessels and the distal nephron of the kidney, or as higher-capacity, lower-affinity transporters, such as CAT-2A, which transport 
these CAAs across the membranes of liver cells. Intracellular arginine concentrations can reach millimolar levels, indicating that physiological arginine concentrations could inhibit DDAH (Teerlink, Luo, Palm, \& Wilcox, 2009). Both ADMA and L-NMMA competitively inhibit all isoforms of NOS, whereas SDMA does not. Because DDAH is mainly present in the liver and kidney, both elimination routes require export from ADMA-generating (endothelial) cells to the plasma. It has been hypothesized that ADMA transport is mediated by the same carrier proteins as those that mediate the transport of other CAA. The major CAA transporters in non-epithelial cells belong to two related protein families: CATs and the system $y+L A A$ transporters $(y+L A T)$. In the kidney and liver, a newly recognized elimination pathway for $A D M A$ is the transamination to $\alpha$-keto- $\delta-(N(G), N(G)$ dimethylguanidino) valeric acid (DMGV) by the enzyme alanine-glyoxylate aminotransferase 2 (AGXT2) expressed in these two organs.

\subsection{Metabolism of ADMA by DDAHs (Figures 4 and 5)}

ADMA is formed ubiquitously in all cells. It can be metabolized intracellularly by DDAH to citrulline and DMA or be exported from the cell to the plasma by the transporters, which are involved in both cellular release and cellular uptake of ADMA. ADMA is metabolized extensively by the kidneys and liver, which are the principal sites of DDAH-1 expression. Some ADMA is excreted by the kidney. DDAHs are highly conserved throughout evolution. Two isoforms of DDAH, which are encoded by genes located on chromosomes 1 (DDAH-1) and 6 (DDAH-2), have been identified. The 2 isoforms have distinct tissue distributions. Both isoforms have been identified within the cardiovascular system (Vallance \& Leiper, 2004).

Although many disease states, including hypertension and diabetes, have been associated with increased plasma levels of ADMA, to date little is known about intracellular levels of ADMA. In most studies, plasma levels of ADMA in humans and rats are in the range of 0.3 to $0.5 \mu \mathrm{mol} / \mathrm{l}$. ADMA is accumulated in cells via CATs, which are widely expressed and account for the high intracellular concentrations of ADMA. Estimates of intracellular ADMA concentrations suggest that levels of ADMA in cells are 10 or 20 times higher than in plasma. Cardounel and al reported that the intracellular levels of ADMA, MMA and L-Arg in freshly isolated brain slices were $10.7 \pm 1.3,5.1 \pm 0.6$ and $94.0 \pm 7.8 \mu \mathrm{mol} / \mathrm{l}$, respectively (Cardounel, et al., 2007; Cardounel, Xia, \& Zweier, 2005). They further showed that the intracellular levels of L-Arg in cultured neurons was constant whereas MMA and ADMA levels decreased by more than $50 \%$ after $24 \mathrm{~h}$ of inhibition of PRMT by Sadenosylhomocysteine. Intracellular ADMA concentrations have been studied in endothelial cells harvested from the carotid artery of the rabbit. The concentrations were 10 times higher in the endothelial cells than in the plasma and were 25 times higher in vessels from rabbits with alloxaninduced diabetes mellitus. It has been demonstrated that intimal hyperplasia after endothelial denudation may be caused, at least partly, through impairment of the ability of endothelial cells to produce/release NO. This process is associated with the accumulation of endogenous L-NMMA and ADMA, which induces a decrease in NO production/release (Masuda, Goto, Tamaoki, \& Azuma, 1999). The intracellular concentrations of ADMA should be very sensitive to changes in the activity or expression of DDAH (Palm, Onozato, Luo, \& Wilcox, 2007). 


\subsection{Metabolism of ADMA and oxidative stress}

Homocysteine oxidizes the sulfhydryl groups in DDAH to form a mixed disulfide, which inactivates the enzyme. DDAHs are very sensitive to oxidative stress because the active site of the enzymes contains a critical sulfhydryl group that is required for the catalytic activity (Teerlink, 2005). It has been reported that pathologic stimuli that induce OS in endothelial cells, such as inflammatory cytokines, hyperhomocysteinemia, hyperglycemia and oxidized low-density lipoprotein (LDL), may reduce DDAH activity (Palm, et al., 2007).

Co-incubation of endothelial cells with ADMA led to a dose-dependent increase in their production of superoxide. This demonstrated the potential for ADMA to increase endothelial ROS (Antoniades, et al., 2009). This establishes a second potential ROS-dependent, amplifying, feed-forward cellular loop, whereby an initial increase in cellular ROS can stimulate the generation of ADMA that further stimulates ROS production (Wilcox, 2012). Increasing bioactive NO by oral administration of nitrate prevented ROS and restored normal levels of ADMA in a rat model of salt-sensitive hypertension (Carlstrom, et al., 2011); dietary nitrate attenuated the level of OS.

As we reported, the inhibition of endothelial NOS by elevated ADMA levels, may lead to the uncoupling of NOS, resulting in a shift from NO production to superoxide production. Several in vitro and in vivo studies on the effect of estrogen, which has anti-oxidant properties on ADMA metabolism, have been conducted. Exposure of endothelial cells to estradiol resulted in an increase in the activity of DDAH and was accompanied by the reduced release of ADMA (Holden, Cartwright, Nussey, \& Whitley, 2003). In addition, a negative association between ADMA and endogenous estrogen levels in women with coronary heart disease has been reported (X. P. Li, et al., 2004).

\section{Cardiovascular and metabolic consequences of increased ADMA concentrations}

\subsection{Endothelial dysfunction and ADMA}

The vascular endothelium plays a key role in cardiovascular physiology and pathophysiology, largely via (NO)-dependent processes. Endothelial dysfunction is defined as the impairment of physiologic endothelium-dependent functions. It occurs in cardiovascular diseases such as atherosclerosis, hypertension, diabetes, hypercholesterolemia, and during normal aging. There are several potential mechanisms for endothelial dysfunction separated into three categories: 1) reduced eNOS expression levels, 2) reduced enzyme activity of eNOS, and 3) rapid removal of NO. As we reported, eNOS requires FAD, FMN, NADPH, and $\mathrm{BH}_{4}$ as cofactors. $\mathrm{BH}_{4}$, whose synthesis is rate-limited by GTP$\mathrm{CH}$, is a particularly important cofactor, because in its absence, electron transport through eNOS can become 'uncoupled,' resulting in the generation of superoxide anion. In summary, NO produced by eNOS may be rapidly inactivated by reaction with superoxide to form peroxynitrite anion. This superoxide can be formed by NOX, or uncoupled eNOS. These conditions may contribute to endothelial dysfunction (H. Liu, et al., 2008). 


\subsection{ADMA and cardiovascular diseases}

\section{ADMA and cardiovascular risk factors}

In some studies, elevated ADMA concentrations have been associated with major cardiovascular risk factors, including hypertension and hypercholesterolemia. Some studies imply that raised ADMA levels may reduce NO bioavailability and accelerate disease progression. In contrast, in other studies, certain cardiovascular risk factors such as age or homocysteine (Hcy) concentrations did not correlate positively with ADMA. Total plasma homocysteine (tHcy) has been considered an independent risk factor for atherosclerotic vascular disease (Duell \& Malinow, 1997) and for acute myocardial infarction (AMI) (Gomes, et al., 2002). Moreover, elevated tHcy concentrations have been associated with a higher risk of recurrent coronary events and death after AMI (Matetzky, et al., 2003).The mechanisms underlying the cardiovascular pathophysiology of hyperhomocysteinemia (hyperHcy) have not yet been completely elucidated, but endothelial dysfunction resulting from OS and the impaired bioavailability of NO is a consistent finding in experimental models. Recently, authors hypothesized that some of the deleterious effects of hyperHcy may involve ADMA-related cardiovascular effects (Boger, 2003). Hcy is derived from the essential amino acid methionine, a methyl donor for arginine methylation. Its level depends on the kinetic properties of enzymes such as S-adenosylmethionine methyltransferases and PRMTs, which participate simultaneously in homocysteine and ADMA synthesis. Consequently, tHcy and ADMA metabolism may be interrelated.

In a recent study, we reported that in aged patients, SDMA and tHcy concentrations correlated positively with ADMA. Age, ADMA, tHcy and creatinine concentrations correlated positively with SDMA levels, while the L-arginine/ ADMA ratio and estimated glomerular filtration rates (eGFR) correlated negatively with SDMA levels. In addition to SDMA, the L-arg/ADMA ratio correlated negatively with age and systolic blood pressure (SBP). ADMA did not correlate significantly with eGFR, and consequently, eGFR was an independent variable in the analysis of ADMA regression. Hcy, a major product of the methylation reaction mediated by PRMTs, could also be related to increased dimethylarginine levels. HyperHcy could increase ADMA levels by reducing DDAH activity via a redoxmediated mechanism and/or directly interfere with DDAH as shown in a cell-free system (Stuhlinger, et al., 2001). Our finding reinforces the importance of quantifying both dimethylarginines in order to investigate their involvement in the development of cardiovascular diseases (Korandji, et al., 2007). Lower ADMA concentrations were associated with smoking, as observed in high-risk elderly men (Teerlink, Nijveldt, de Jong, \& van Leeuwen, 2002).

\section{ADMA hypertension and heart failure}

The first evidence that ADMA may play a role in disease came from studies showing that plasma ADMA concentrations were elevated in patients with end-stage renal failure and that this elevation correlated with reduced NO production (Vallance \& Leiper, 2004). Now it has been demonstrated that in various pathological states such as hypertension, plasma levels of ADMA may be increased and lead to the inhibition of NO synthesis and endothelial dysfunction. The inhibition of ADMA synthesis or the intensification of ADMA metabolism might indirectly lower ADMA. The treatment of hypertension with drugs directed against the angiotensin system reduced circulating levels of ADMA. 
The addition of the angiotensin AT1-receptor blocker telmisartan to tissue slices from rat kidneys incubated with angiotensin II increased DDAH-1 protein expression (Onozato, et al., 2008).

Plasma levels of ADMA and SDMA were elevated in patients with end-stage renal failure, due in part to decreased renal elimination (Vallance, et al., 1992). Angiotensin converting enzyme inhibitors or angiotensin AT1 receptor antagonists, and also beta-adrenoreceptor antagonists are associated with decreased ADMA levels (Kawata, et al., 2009; Pasini, et al., 2008). The relationships between angiotensin II, NO production and ADMA levels are complex. Angiotensin II (Ang II) can generate ROS in blood vessels by the activation of NOX. Patients with early hypertension or kidney disease have elevated plasma levels of ADMA and markers of ROS, which may contribute to endothelial dysfunction and subsequent cardiovascular or renal events. Although increased ADMA occurs in several conditions associated with ROS. The membrane protein p22phox is a critical component of NOX (Ushio-Fukai, Zafari, Fukui, Ishizaka, \& Griendling, 1996). Smooth muscle specific overexpression of p22phox in mice increased aortic p22phox and NOX-1 proteins and increased $\mathrm{O}_{2}{ }^{-}$and $\mathrm{H}_{2} \mathrm{O}_{2}$ generation, whereas knockdown of p22phox in vivo reduced the protein expression for NOX-1, -2, and -4 . The incubation of rat preglomerular vascular smooth muscle cells (PGVSMCs) with angiotensin II doubled the activity of NOX but decreased the activities of DDAHs by $35 \%$ and of cationic amino acid transport by $20 \%$ and doubled cellular (but not medium) ADMA concentrations. The concentration of ADMA in PGVSMCs was 27 times greater than in the medium. The calculated intracellular concentration of ADMA was $6 \mu \mathrm{mol} / \mathrm{L}$ (Luo, et al., 2010).

There is very little information regarding the effects of hypertension on intracellular levels of ADMA. A correlation between the levels of plasma ADMA and arterial pressure has been reported in patients with uncomplicated early hypertension (D. Wang, Strandgaard, Iversen, \& Wilcox, 2009).Thus, elevated plasma levels of ADMA could play some role in the development of hypertension (Jacobi, et al., 2008). Elevated plasma levels of ADMA have been reported in hypertensive children (Goonasekera, et al., 1997) and in elderly hypertensive patients (Kielstein, et al., 2003). Plasma levels of ADMA are higher in hypertensive than in normotensive subjects, whereas plasma levels of SDMA are similar. This could indicate reduced intracellular ADMA metabolism by DDAH (65). However, other studies have reached different conclusions. Significant inverse correlations or no correlations at all between plasma levels of ADMA and diastolic blood pressure have been reported (Boger, Sullivan, et al., 2009; Delles, Schneider, John, Gekle, \& Schmieder, 2002).

Recently, it has been demonstrated that an elevated circulating level of ADMA is a strong independent predictor of short-term and long-term mortality in patients with decompensated coronary heart failure (CHF) and reduced left ventricular ejection fraction. ADMA levels on presentation may enable enhanced risk stratification in this setting (Zairis, et al., 2012). The accumulation of ADMA in patients with CHF may be attributed to the inhibition of DDAH activity, the main route of ADMA degradation, which is believed to occur due to the heightened oxidative stress that accompanies CHF (Searles, 2002). Taken together, most available data suggest that elevated circulating levels of ADMA can result in, or result from, hypertension and may increase the risk of adverse cardiovascular events (Teerlink, et al., 2009). 


\subsection{ADMA and mortality}

There is little information on the relevance of ADMA as a marker of morbidity and mortality in population-based cohorts. The reason for this is that very large populations need to be studied, and analytical techniques have only recently become available. The aim of a study in our laboratory in a prospective cohort of patients with acute MI was to identify the determinants of ADMA levels and to analyze the predictive value of ADMA on mortality in the long term (Zeller, et al., 2008). Our findings suggest that ADMA levels have a predictive value for long-term mortality after $\mathrm{MI}$, beyond that obtained with baseline determinants of prognosis. In Finnish middle-aged men elevated levels of plasma ADMA were initially shown to be associated with the risk of cardiovascular (CV) events (Valkonen, et al., 2001). In a large cohort of patients with documented CV disease, ADMA levels were independently associated with cardiovascular death in the long term, with a $27 \%$ increase in risk for each increment of 1-SD in baseline ADMA values (Schnabel, et al., 2005).

Plasma levels of ADMA, SDMA, and L-arginine were evaluated in 3320 participants of the Framingham Offspring Study (Boger, Maas, Schulze, \& Schwedhelm, 2009; Boger, Sullivan, et al., 2009). The Framingham Offspring Study is a prospective, population based cohort comprising the offspring of subjects included in the original Framingham Heart Study cohort in the 1950s (Kannel, Feinleib, McNamara, Garrison, \& Castelli, 1979). Mean age was 59 years, and our sample comprised 364 subjects with prevalent cardiovascular disease and 372 subjects with diabetes mellitus at the time of sample collection. A positive association was noted between ADMA levels and total mortality, which remained significant after adjustment for a variety of traditional parameters. Whilst SDMA and L-arginine levels were not associated with mortality, the L-arginine/ADMA ratio showed a significant, inverse association with risk.

\subsection{Metabolic parameters and ADMA}

\section{Diabetes}

The predictive value of plasma ADMA concentrations is of fundamental importance in subjects with diabetes, in whom the risk of heart disease and coronary events remains high. In diabetics, it has been suggested that chronic modifications in ADMA levels mediate endothelial dysfunction and increase the associated risk of developing CV diseases. With respect to diabetes, as plasma insulin and glucose vary over any 24-h period, it is essential to understand how acute fluctuations might modulate plasma ADMA levels. Plasma concentrations of ADMA reflect the balance between the rate of production and the rate of removal. In acute circumstances, the rate of ADMA production is almost exclusively dependent on the rate of proteolysis, while the rate of removal is dependent on DDAH activity. During the post-prandial period, there is a significant increase in plasma insulin levels, arising from compensatory increases in the insulin infusion rates associated with rises in glucose levels. In this context, it has been reported that insulin plays a significant role in modulating plasma ADMA concentrations, but it is probable that differences in plasma insulin levels in insulin-resistant subjects might play the main role in influencing the rate of synthesis and/or catabolism of methylarginines (Marcovecchio, Widmer, Dunger, \& Dalton, 2008). Several studies have reported elevated plasma levels of ADMA in patients with type I and II diabetes mellitus (DM), prior to gestational diabetes. In contrast, decreased plasma levels of ADMA have been reported in patients 
with type 2 diabetes (Abbasi, et al., 2001; Paiva, et al., 2003). This may be related to the differential effects of glucose and insulin on the metabolism of ADMA. High glucose levels may impair DDAH activity by inducing oxidative stress leading to increased intracellular levels of ADMA (Ellger, et al., 2008). On the other hand, reduced DDAH activity has been reported in a number of medical conditions, especially when kidney and liver function are impaired. Therefore, reduced DDAH activity and thereby increased ADMA levels may result from organ failure (Siroen, et al., 2006).

As we reported, in some studies, serum levels of ADMA are elevated in both type 1 and type 2 diabetes, but a number of studies have also found a reduction in serum ADMA associated with these pathologies. As we previously reported, some organs or isolated cells may use transporters to export ADMA to the plasma compartment, whereas other organs, such as the liver and kidney, may incorporate ADMA from the circulation, and subsequently degrade it by DDAH. In this context, most available data suggest that endothelial cells have an important function. Interestingly, levels of ADMA in endothelial cells from the rabbit carotid artery were 10 times higher than plasma levels and increased further from 5.0 to $12.1 \mu \mathrm{mol} / \mathrm{l}$ after the induction of alloxan-induced diabetes (Masuda, et al., 1999). Metformin lowered the blood glucose concentrations in diabetic patients and simultaneously reduced the circulating levels of ADMA, although there was no relationship between the decrease in ADMA concentration and the improvement in glycemic control (Asagami, et al., 2002). Insulinopenic diabetic rats had a 50\% increase in renal angiotensin II concentrations concomitant with increased plasma levels of ADMA (Onozato, et al., 2008). Interestingly, the pathways that generate and metabolize ADMA in the kidneys of these rats were altered by an angiotensin AT1- receptor blocker. A reduction in circulating levels of ADMA by an angiotensin AT1receptor blocker in a model of type-1diabetes may be a result of both decreased ADMA synthesis by PRMT-1 and increased ADMA metabolism by DDAH-1. There is little information regarding the effects of the stimulation of angiotensin receptors on PRMTs and DDAH-1 activity and the intracellular levels of ADMA. Among these processes, the trans-cellular transport of methylarginine may be of particular importance in chronic illness (Teerlink, et al., 2009).

\section{Metabolic syndrome and Insulin resistance}

The metabolic syndrome (MS) is associated with abdominal obesity, blood lipid disorders, inflammation, insulin resistance, and increased risk of developing cardiovascular diseases and diabetes. There is growing evidence that endothelial dysfunction is a component of the MS and that endothelial dysfunction is caused by the reduced bioavailability of NO in the vascular wall. Evidence indicates that endogenous NOS inhibitors such as ADMA may be causally related to this process (Palomo, et al., 2011). In this field of epidemiological and clinical studies it has been demonstrated that a high-fat diet induces insulin resistance and that endogenous NOS inhibitors emerged as a marker associated with cardiovascular risk. Most studies suggest that increased dietary fat causes whole-body and regional (liver, adipose tissue, muscle) insulin resistance in both animals and humans. NO regulates metabolic lipid and carbohydrate metabolism. Glucose metabolism is enhanced by NO, in part by upregulation of the GLUT transporter, and possibly by enhanced vascular delivery of glucose to insulin-sensitive tissues (Andersson, et al., 1999; Sartori \& Scherrer, 1999). Insulin resistance, as well as hypertension and hyperglycemia, is a central component of metabolic syndrome, and has been associated with an increase in cardiovascular risk (Reaven, 2003). Some 
studies suggest a strong association between insulin resistance and endothelial dysfunction. The relationship between ADMA metabolism and oxidative stress has not yet been clearly demonstrated, in particular during the development of an insulin resistance syndrome. The aim of a study in our laboratory (Korandji, et al., 2011) was to study the nature of and the relationship between different metabolic parameters involved in the regulation of oxidative stress in the fructose-fed rat, an experimental model of cardiovascular complications related to metabolic syndrome. Unlike glucose, fructose does not directly stimulate insulin secretion because pancreatic beta-cells have very low levels of the fructose transporter GLUT5 (Sato, et al., 1996). Our study showed that the high fructose diet was associated with the development of metabolic disorders such as hypertension, hyperglycemia and dyslipidemia, all of which are components of insulin resistance syndrome. Our work also showed that plasma levels of ADMA increased only after the rise in arterial pressure and glycemia. We found that a long-term fructose-rich diet was associated with an increase in oxidative stress. The increase in vascular OS markers was noted from the second week of treatment onwards. In contrast, the increase in NOX activity occurred much later. Furthermore, our findings demonstrated that the high-fructose diet diminished aortic endothelium-dependent relaxation in the aortic wall. Our results are in accordance with a study (Oron-Herman, et al., 2008) which indicated that fructose-fed Sprague-Dawley rats manifested major characteristics of human metabolic syndrome. Although fructose has been recommended to diabetic patients, there are concerns that fructose may contribute to the development of metabolic syndrome (Bray, Nielsen, \& Popkin, 2004). Our work showed that the increase in plasma levels of ADMA in the fructose-fed rats occurred after 2 weeks of diet and after the increase in glycemia. Considering the results of our study, the interaction between hyperglycemia and the increase in plasma ADMA could be explained, at least in part, by the rise in protein catabolism associated with the development of oxidative stress. In hyperglycemia ( $25.5 \mathrm{mmol} / \mathrm{L})$, DDAH activity was reduced by about $20 \%$ and significantly correlated with increasing ADMA (Lin, et al., 2002). Therefore, in our experimental conditions of hyperglycemia ( $9 \mathrm{mmol} / \mathrm{L})$, the increase in circulating ADMA could be partly linked to the inhibition of enzymes such as DDAH.

\section{Atherosclerosis}

Atherosclerosis is driven by biochemical, cellular, and hemodynamic forces in the vessel wall that cause vascular injury, ultimately leading to endothelial dysfunction, cellular proliferation, recruitment of inflammatory cells, and the accumulation of oxidized LDL (Libby, 2008). The decreased bioavailability of endothelial NO is thought to be associated with the development of atherosclerosis (Capettini, Cortes, Silva, Alvarez-Leite, \& Lemos, 2011). Most articles that have explored the relationship have found increased ADMA or an increased ADMA/L-arginine ratio in patients with high cholesterol (Boger, 2003). A high concentration of plasma ADMA has been associated with several risk factors for atherosclerosis and a high risk of acute coronary events (Miyazaki, et al., 1999; Valkonen, et al., 2001). However, the underlying mechanisms of endothelial eNOS/NO dysfunction in atherosclerosis have been intensively studied and various mechanisms responsible for decreased endothelial NO bioactivity under the disease condition have been suggested. Several clinical genetic studies identified different common eNOS gene polymorphisms that might be associated with atherosclerotic coronary artery disease. Among the eNOS polymorphisms so far identified, G894T substitution within exon 7 resulting in the conversion of glutamate to aspartate at position 298 (Glu298Asp variant) is the most frequently studied and has been reported to be associated with 
atherosclerotic coronary artery disease in two independent cohorts recruited from the Cambridge Heart AntiOxidant Studies (CHAOS-I and CHAOS-II) (Hingorani, et al., 1999). Several biochemical mechanisms are thought to be involved in eNOS uncoupling in atherosclerosis. These include $\mathrm{BH}_{4}$ deficiency and an increase in endogenous ADMA, L-arginine deficiency and oxidative stress. Most researchers believe that co-factor $\mathrm{BH}_{4}$ deficiency is the mechanism involved in eNOS uncoupling, which plays a role in endothelial dysfunction under various pathological conditions, including atherosclerosis (Alp \& Channon, 2004). The relationship between Hcy and ADMA is of interest because there are many potential interactions. Hcy can inhibit DDAH activity, possibly by an interaction with the critical cysteine residue in the active site of the enzyme. (Vallance \& Leiper, 2004).

In conclusion, extensive clinical data support the notion that reducing ADMA levels may provide a novel therapeutic approach in the treatment of cardiovascular disease, as reduced NO production has been implicated in the development of cardiovascular disease.

\section{NO synthases/ADMA - Therapeutic targets}

Due to the clinical properties of endothelial NO, the eNOS enzyme is an interesting target for the prevention or treatment of cardiovascular diseases. Oxidative stress is likely to be the main cause of oxidation of the essential NOS cofactor, $\mathrm{BH}_{4}$. $\mathrm{A}$ lack of $\mathrm{BH}_{4}$ leads to eNOS uncoupling (i.e., uncoupling of oxygen reduction from NO synthesis in eNOS). In this context, eNOS modulators and their therapeutic effects are discussed in this review: (1) Substrate availability: L-Arginine (2) eNOS transcription enhancers (3) treatment with $\mathrm{BH}_{4}(4)$ treatment with folic acid (5) treatment with Transresveratrol (6) statins (7) drugs that interfere with the renin-angiotensin-aldosterone system (8) modulation of DDAH and PRMT activities (9) selective NOX inhibitors.

\subsection{Substrate availability: L-Arginine}

Under physiological conditions, the intracellular concentration of L-Arg is generally high enough to be in excess of the $\mathrm{Km}$ for eNOS. However, cardiovascular diseases associated with oxidative stress have been linked with reduced L-Arg transport. In our laboratory, we investigated the role of NOS modulation on 1) the evolution of functional parameters and the level of post-ischemic recovery and 2) the amount of free radical species released during a sequence of global myocardial ischemia and reperfusion, using L-arginine as a substrate or NG-nitro-L-arginine methyl ester (L-NAME) as an inhibitor, in comparison with their D-specific enantiomers (C. Vergely, Perrin-Sarrado, Clermont, \& Rochette, 2002). Under our experimental conditions, there was no significant difference in cardiac function between hearts treated with D- or L-Arg, despite a slight increase in post-ischemic peak myocardial function and a decrease in heart rate. Many authors have described the beneficial effects of L-Arg treatment on the recovery of myocardial function during reperfusion (Padilla, et al., 2000) whereas others have shown a harmful effect of L-Arg (Mori, Haramaki, Ikeda, \& Imaizumi, 1998). These discrepancies can be attributed to different experimental models but also to the critical timing of the administration of this amino acid. Moreover, it has been reported that there is a species 
difference in the effectiveness of endogenous NO to protect hearts against reperfusion-induced dysfunction (Pabla \& Curtis, 2007). All these data are not consistent with the notion that NO is a ubiquitous endogenous cardioprotector.

As previously mentioned, one of the main factors in the regulation of L-Arg transporters is substrate availability. It has been reported that the administration of exogenous L-Arg restores NO bioavailability (Candipan, Wang, Buitrago, Tsao, \& Cooke, 1996) but it has not been possible to demonstrate, in all studies, that L-Arg supplementation improved endothelial function in cardiovascular disease such as heart failure or hypertension (Chin-Dusting, et al., 2007). The results have demonstrated varying positive effects on endothelial function, probably due to substantial variations in the timing and doses of L-Arg. Clinical studies with L-Arg have also shown inconsistent effects on endothelial function. In some clinical studies, acute and subacute L-Arg administration improved NO-dependent vasodilatation in patients with coronary artery disease or hypercholesterolemia. In contrast, the VINTAGE MI study demonstrated that in patients assigned to receive L-Arg (goal dose of $3 \mathrm{~g} 3$ times a day) or a matching placebo for 6 months, L-Arg did not improve vascular stiffness measurements or ejection fraction and may have been associated with higher post-infarction mortality (Schulman, et al., 2006). The conclusion of this study was that L-Argtherapy should not be given to patients following myocardial infarction. It neither alters non-invasive measurements of vascular stiffness nor improves left ventricular function. L-Arg therapy in older patients with diffuse atherosclerosis may worsen clinical outcomes. In addition to these negatives results, one can postulate that in these pathologies it is not clear that endothelial dysfunction was related to L-Arg deficiency.

\section{2. eNOS transcription enhancers}

A primary screening of chemical libraries for compounds that increase eNOS transcription yielded two small-molecular-weight compounds with related structures, namely AVE9488 (earlier designation C2431) and AVE3085 (Related structures, 4-fluoro-Nindan- 2-yl-benzamide (CAS no. 291756-32-6; empirical formula C16H14FNO; AVE9488) and 2,2-difluoro-benzo[1,3]dioxole-5carboxylic acid indan-2-ylamide (CAS no. 450348-85-3; empirical formula C17H13F2NO3; AVE3085). AVE9488 and AVE3085 increase endothelial NO production by the simultaneous up-regulation of eNOS expression and reversal of eNOS uncoupling (Wohlfart, et al., 2008). These compounds protect the heart against ischemia/reperfusion injury, and improve left ventricular remodeling after myocardial infarction (Fraccarollo, et al., 2008; Frantz, et al., 2009). Another analogue, AVE3085, improves endothelial function and reduces blood pressure in spontaneously hypertensive rats (SHRs). AVE3085 ameliorates endothelial dysfunction in $\mathrm{db} / \mathrm{db}$ mice through increased NO bioavailability, which reduces oxidative stress in the vascular wall. Targeting eNOS and NO production may be a promising approach to combat diabetic vasculopathy (Cheang, et al., 2011). Recently, it has been demonstrated that AVE3085 restored impaired endothelial function in spontaneously hypertensive rats by the upregulated expression of eNOS protein and mRNA, enhanced eNOS phosphorylation, and decreased formation of nitrotyrosine (Yang, et al., 2011). The peroxisome proliferator-activated receptor (PPAR) alpha agonist AVE8134 had not only a direct effect on cardiomyocyte hypertrophy, but also an indirect effect via monocyte signaling and increased endothelial NO production (Linz, et al., 2009). 


\subsection{Treatment with $\mathrm{BH}_{4}$}

The attention in the field of $\mathrm{BH}_{4}$ has shifted from structural and metabolic-molecular aspects to homoeostasis of the $\mathrm{BH}_{4}$ system and the pathophysiology of $\mathrm{BH}_{4}$ deficiency, with greater emphasis on the in vivo effects on NOS decoupling (Werner, et al., 2011). The importance of $\mathrm{BH}_{4}$ as a critical regulator of eNOS function suggests that $\mathrm{BH}_{4}$ may be a rational therapeutic target in vascular disease states (Crabtree \& Channon, 2011). More than 400 reports have been published to date on studies trying to ameliorate vascular and heart dysfunctions by treatment with $\mathrm{BH}_{4}$ (Katusic, d'Uscio, \& Nath, 2009; Schulz, Jansen, Wenzel, Daiber, \& Munzel, 2008). Recently, new evidence has suggested a beneficial role of iNOS recoupling as a possible treatment for ischemia/reperfusion injury and metabolic disease in diabetes. Supplementation with $\mathrm{BH}_{4}$ or its physiological precursor, sepiapterin, improved endothelial function in vessels from animal models of hypercholesterolemia and diabetes (Alp \& Channon, 2004). In the light of experimental findings, modulation of the arginine-NO pathway by $\mathrm{BH}_{4}$ and arginine has been developed in clinical studies, the aim being to improve vascular insulin resistance in obesity and diabetes. A clinical study has shown that concomitant intra-arterial infusion of $\mathrm{BH}_{4}$ in type 2 diabetic patients improved endothelium-dependent vasodilation, demonstrating the therapeutic potential of upregulating $\mathrm{BH}_{4}$ bioavailability (Heitzer, Krohn, Albers, \& Meinertz, 2000). But it is now suggested that these results may have limited long-term benefits. Chronic $\mathrm{BH}_{4}$ supplementation may result in the accumulation of $\mathrm{BH}_{2}$ in the endothelium, which will induce eNOS uncoupling (Crabtree, et al., 2008). Many small clinical trials have supported the benefits of $\mathrm{BH}_{4}$ treatment in vascular disorders and in particular endothelial disease (Katusic, et al., 2009). With the development of synthetic sapropterin hydrochloride $\left(6 \mathrm{R}-\mathrm{BH}_{4}, \mathrm{Kuvan}^{\circledR}\right)$, larger trials with an FDAapproved formulation became feasible, and raised hopes that similar efficacy would be observed. 6R$\mathrm{BH}_{4}$ was studied in a number of phase I-II clinical trials for diseases (Moens, et al., 2011; Moens, et al., 2008). There is now a need to determine the dose-response relationship between $\mathrm{BH}_{4}$ and its effect on oxidative stress in models of diabetes, because of the tight stoichiometric relationship between eNOS and $\mathrm{BH}_{4}$, with possible subsequent uncoupling.

\subsection{Treatment with folic acid}

Folate (also known as vitamin $B_{9}$ ) is a generic term for a group of water-soluble compounds composed of a pteridine ring and glutamic acid. Folate is essential for DNA synthesis, repair and methylation, as well as a variety of metabolic processes. Adequate consumption of dietary folates is considered to lower the risk of cardiovascular disease, in particular by improving endothelial function associated with an action on Hcy metabolism. HyperHcy has emerged as an independent risk factor for atherosclerosis and vascular disease. One important function of folic acid is to reduce plasma Hcy, and folic acid has direct and indirect superoxide scavenging actions. It has been reported that folic acid increased the vascular bioavaibility of $\mathrm{BH}_{4}$ and subsequently reduced eNOS-derived ROS generation (Antoniades, et al., 2006). But there was discordance concerning the epidemiology of Hcy, and the results of the clinical trials completed to date are similar to those reported for antioxidant vitamins. Combined daily administration of folic acid, vitamin $B_{6}$, and vitamin $B_{12}$ for five years had no beneficial effects on major vascular events in a high-risk population with vascular disease (Lonn, et al., 2006). 


\subsection{Treatment with Trans-resveratrol}

Resveratrol (3,5,4'-trihydroxy-trans-stilbene) is a polyphenol phytoalexin present in a variety of plant species and has been implicated in the health benefits of red wine. In animal studies, resveratrol has been shown to exhibit various biological effects probably attributable to the fact it has many molecular targets including a number of signaling pathways. These effects may be beneficial in many disorders, particularly in diseases where oxidative stress plays an important role. While resveratrol does not function as a strong in vitro scavenger of ROS, it does function as a potent in vivo antioxidant, which probably arises from its ability to increase NO synthesis, which in turn acts as an antioxidant (Svajger \& Jeras, 2012; Turan, Tuncay, \& Vassort, 2012). As already noted, the reversal of eNOS uncoupling might be a novel therapeutic approach. It has been reported that the treatment of apolipoprotein $E$ knockout (ApoE-KO) mice with resveratrol resulted in the up-regulation of superoxide dismutase (SOD) isoforms (SOD1-SOD3), glutathione peroxidase 1 (GPx1), and catalase and the down-regulation of NOX2 and NOX4 in the hearts of these mice. In parallel, the cardiac expression of GTP-CH was enhanced by resveratrol and accompanied by an elevation in $\mathrm{BH}_{4}$ levels. Resveratrol treatment resulted in a reversal of eNOS uncoupling (Xia, et al., 2010). There is a wealth of evidence attesting to the fact that resveratrol controls different endogenous systems that maintain antioxidant enzymes and thus play a key role in cellular defense against free radical damage. At low concentrations resveratrol may act as an antioxidant by scavenging ROS, whereas at higher doses it may act as a pro-oxidant (Miura, Muraoka, Ikeda, Watanabe, \& Fujimoto, 2000). Furthermore, recent results show that the effect of resveratrol on membrane fluidity may also be related to its protective effects in various pathological processes (Brittes, Lucio, Nunes, Lima, \& Reis, 2010).

\subsection{Treatments with Statins}

Statins are used clinically for their potent plasma lipid-lowering effect, but they have also been shown to possess other significant antiatherogenic properties. Among their pleiotropic effects, statins have antioxidant and anti-inflammatory properties. In our laboratory, we evaluated in normotensive (WKY) and in spontaneously hypertensive rats (SHR) the effect of rosuvastatin (ROSU) treatment on (1) plasma inflammation markers and ADMA levels, (2) ROS generated by circulating leukocytes and (3) vascular oxidative stress and tissue inflammation markers. Plasma cytokines were higher in SHR than in WKY, except for IL-4, which was lower in SHR than in WKY. SHR monocytes exhibited higher production of ROS than did WKY monocytes. In experimental conditions, ROSU did not modify plasma cholesterol levels in SHR but attenuated the increase in systolic blood pressure. In SHR only, ROSU lessened pro-inflammatory cytokines and ADMA levels, increased IL-4 and reduced ROS production in circulating monocytes. These results demonstrate the beneficial effects of ROSU in SHR, independently of any lowering of cholesterol levels (Sicard, et al., 2008).

Emerging data suggest that statins have a beneficial effect on telomere biology, which possibly contributes to the beneficial effects of statin therapy in relation to its action on oxidative stress. Recently, we tested the hypothesis that leukocyte telomere length (LTL) could be affected by statin treatment and related to the transcript levels of FOS and OGG1, as new biomarkers of TL associated- 
oxidative stress and inflammation. 8-Oxoguanine DNA glycosylase (OGG1) is involved in a base excision repair pathway that has recently been shown to be active after telomeric DNA oxidative damage, and Finkel-Biskis-Jinkins osteosarcoma (FOS), a ROS-induced transcription factor, is specifically involved in atherosclerosis-associated inflammation. Our observational study showed that statin therapy was associated with longer LTL. These data bring to light opportunities to identify new targets for early primary preventive treatment strategies. Moreover, our study showed that FOS and OGG1 were new relevant biomarkers of LTL (Saliques, et al., 2011).

Statins have been reported to increase $\mathrm{BH}_{4}$ levels in vascular endothelial cells by potentiating GTP-CH gene expression and $\mathrm{BH}_{4}$ synthesis, thereby increasing $\mathrm{NO}$ production and preventing relative shortages of $\mathrm{BH}_{4}$ (Martinez-Gonzalez, Raposo, Rodriguez, \& Badimon, 2001). A recent study demonstrated that fluvastatin upregulated eNOS activity via enhancement of its phosphorylation and expression and via an increase in $\mathrm{BH}_{4}$ in vascular endothelial cells (Aoki, et al., 2012). Fluvastatin stimulates increased eNOS phosphorylation at Ser-1177 and Ser-633 through the PI3-kinase/Akt and PKA pathways, respectively. Finally, fluvastatin may increase eNOS activity by inducing eNOS, as well as by increasing the amount of available $\mathrm{BH}_{4}$.

\subsection{Drugs that interfere with the renin-angiotensin-aldosterone system}

Angiotensin II is a potent NOX activator and is implicated in the pathogenesis and progression of various cardiovascular disorders. Therefore, increased production of angiotensin II is an attractive hypothesis to explain the age-related increase in vascular dysfunction. We demonstrated that agerelated cardiac vulnerability and vascular changes are accompanied by increased superoxide production. This increase in superoxide production, particularly at the vascular level, is due to the increased expression of endothelial gp91phox-containing NOX associated with an increase in the expression of endothelial angiotensin AT1 (Oudot, et al., 2006). One study reported the effects of cotreatment with aliskiren (a renin inhibitor) and valsartan (angiotensin II receptor blocker) in Watanabe heritable hyperlipidemic rabbits treated with vehicle (control), aliskiren, valsartan, or aliskiren plus valsartan for 8 weeks. Both vascular superoxide and peroxynitrite levels were significantly lower in the aliskiren+valsartan group than in the aliskiren or valsartan group. The highest $\mathrm{BH}_{4}$ levels were observed after aliskiren+valsartan cotreatment (Imanishi, et al., 2008). It was also reported that the inhibitory effects of aliskiren on peroxynitrite and superoxide production would, at least in part, result in potentiated NO bioavailability through the suppression of NO breakdown. Second, this study showed that aliskiren treatment significantly upregulated eNOS phosphorylation, which is crucial to eNOS activity, and Akt phosphorylation. The Hsp90-Akt-eNOS complex is believed to be integral to eNOS-dependent NO production (Pi \& Patterson, 2008).

It has been reported that in diabetic rat kidneys, NOX and uncoupled eNOS were major sources of glomerular superoxide (Satoh, et al., 2005). In the glomeruli of rats with streptozotocin-induced diabetes, the administration of losartan improved NOS function, as determined by increased glomerular NO production and decreased glomerular ROS production. These results suggest that angiotensin receptor blockers can improve uncoupled NOS in the diabetic glomeruli (Satoh, et al., 2008). Moreover, this study confirmed that GTP-CH protein expression levels were decreased in diabetic glomeruli and attenuated by losartan treatment without affecting mRNA expression. Losartan may therefore influence GTP-CH activity. It had been reported that diabetic hyperglycemia 
activated the $26 \mathrm{~S}$ proteasome via peroxynitrite and resulted in the ubiquitination and degradation of GTP-CH, thus limiting de novo $\mathrm{BH}_{4}$ synthesis (Xu, et al., 2007).

Aldosterone antagonism has been used clinically for many years to treat conditions often related to oxidative stress. It has been established that blocking mineralocorticoid receptors by spironolactone has significant effects on the inhibition of oxidative stress, predominantly through the inhibition of NOXs (Virdis, et al., 2002). Another investigation, performed on male Wistar rats demonstrated that eplerenone, a mineralocorticoid receptor antagonist, improved endothelial dysfunction and inhibited expression of the NOX subunit p22phox in the early post-myocardial infarction phase (Sartorio, et al., 2007). The potential for renin-angiotensin-aldosterone inhibition in the regulation of NOXs is now corroborated by numerous studies in cardiovascular diseases. AT1 receptor blockers can be considered one of the most potent drugs currently available for inhibiting the activation of NOXs (Luther \& Brown, 2011).

\subsection{Modulation of DDAH or PRMT activity as a therapeutic target}

\section{DDAH activity}

DDAH modulation is a promising pharmaceutical strategy to indirectly affect NO formation by modifying $\mathrm{N}$-methylated l-arginine levels. Modulation of DDAH could therefore be used to restore physiological NO signaling. Several studies have demonstrated that the overexpression of DDAH or increased transcription of genes that encode DDAH isoforms are sufficient to reduce ADMA levels and increase NO production. For example, the treatment of vascular smooth muscle cells with IL-1 $\beta$ induces expression of DDAH1 with a concomitant reduction in ADMA levels (Ueda, et al., 2003). Control of NO levels by manipulating the ADMA-DDAH-NO pathway is a significant avenue for the potential early treatment of peripheral arterial disease (PAD). In PAD, the local upregulation of DDAH in order to reduce ADMA levels within the ischemic muscle may be beneficial in a number of ways: it may improve microvascular function and local tissue perfusion (Williams, et al., 2012). As we previously reported, the accumulation of ROS in hyperglycemia may partly contribute to decreased DDAH activity and DDAH expression. This hypothesis is supported by the observation that antioxidants such as NAC can restore DDAH activity and increase expression of DDAH II in highglucose conditions, with a corresponding reduction in mean ADMA levels. In this context, various strategies have been explored to achieve therapeutic NO production. A number of clinically utilized compounds increase DDAH activity and increase eNOS expression (Leiper \& Nandi, 2011). Most of the pharmacological and biological agents, such as hormones (insulin, oestradiol, adiponectin) and vitamins (vitamins $A$ and $E$ ), which enhance or restore DDAH activity/expression, exert further salutary effects on vascular homeostasis (Wadham \& Mangoni, 2009). Among the pharmacological agents, there are lipid lowering drugs (statins), antihypertensive (nebivolol) and hypoglycemic agents (aminoguanidine). There is now an extensive search for agents to modulate the expression of different DDAH isoforms and their polymorphism. A study in this field in a large Australian cohort of individuals with type 2 diabetes reported that serum ADMA levels were influenced by common single nucleotide polymorphisms (SNPs) in DDAH1 and DDAH2 genes (Abhary, et al., 2010). This study found that genetic variations in the DDAH1 and DDAH2 genes were significantly associated with serum ADMA levels in patients with type 2 diabetes. More recently, new findings suggest that the rs9267551 polymorphism in the DDAH2 gene through its effects on ADMA levels may impair eNOS 
activity and NO production resulting in impaired insulin-stimulated glucose disposal predominantly in skeletal muscle (Andreozzi, et al., 2012). The relationships between ADMA, NO, DDAH enzymes and diabetes are complex and important differences exist in the genetic regulation of serum levels of ADMA in the different forms of diabetes.

\section{PRMT activity}

There are currently nine proteins in humans known to possess PRMT activity. Arginine methylation is known to affect many mechanisms, mostly involving the modulation of processes involving nucleic acids (Krause, et al., 2007). Aberrant regulation of PRMT activity is associated with various pathological states such as cancer and cardiovascular disorders. Since PRMT-knockout mice display different phenotypes and severities, it is generally considered that PRMTs play a role in embryogenesis and adult tissue homeostasis. The development and application of small-molecule PRMT inhibitors (named arginine methyltransferase inhibitors: AMIs) or activators will provide new fields for therapeutic discovery (Cha \& Jho, 2012). Inhibitors of the PRMT have been reported; most are not specific to PRMT but rather act on a wide variety of $S$-adenosylmethionine (AdoMet)dependent methyltransferases. One example is S-adenosyl-homocysteine. Another compound that potently inhibits the arginine methylation of maltose-binding protein is a natural streptomycin antibiotic called sinefungin. A series of polyaromatic compounds, discovered by the drug-screening process, are specific to PRMT (Cheng, et al., 2004). Other PRMT inhibitors have been screened (Heinke, et al., 2009). Two potent compounds, A9 and A36, which exhibit inhibitory effects, were studied; they were found to be more efficacious than the previously reported PRMT1 inhibitor, AMI1. A9 significantly inhibits the proliferation of castrate-resistant prostate cancer cells. In addition, A9 may be a potential inhibitor against advanced hormone-independent cancers, and the work will provide clues for the future development of specific compounds that block the interaction of PRMTs with their targets (J. Wang, et al., 2012). An alternative way to inhibit PRMT-mediated arginine methylation was recently reported. Rather than developing inhibitors of the enzyme active site, Feng and coworkers developed small molecules that target the substrate to block PRMTs (Feng, Li, Wang, \& Zheng, 2010). Naphthalene-sulfonate (NS) analogs have also been discovered. NS-1 and similar structural analogs including AMI-1 were found to interact directly with the substrate, not the enzyme, to block PRMT1-mediated arginine methylation (Yost, Korboukh, Liu, Gao, \& Jin, 2011). The lack of selective inhibitors is a key obstacle currently limiting research efforts to define the functions of individual PRMT isoforms.

Aberrant expression of PRMTs has been identified in human cancers, and PRMTs are considered therapeutic targets. A recent study showed that PRMT1 and PRMT6 expression is significantly upregulated in various types of cancer, including non-small-cell lung cancer, small-cell lung cancer, bladder cancer and breast cancer. Knockdown of PRMT1 or PRMT6 with specific siRNAs was shown to significantly suppress the growth of bladder and lung cancer cell lines (Yoshimatsu, et al., 2011). Recent studies showed that dysregulation of PRMT5 methyltransferase activity can be a risk factor for neoplastic transformation (F. Liu, et al., 2011). Knockdown of PRMT5 reduces the proliferation of transformed lymphoid cancer cell lines (Pal, et al., 2007). In cardiovascular disease, increased PRMT-1 protein expression localized to the left atrium during atrial fibrillation (AF) in dogs has been reported. Plasma levels of ADMA were significantly higher in the AF group than in the control group $(\mathrm{H}$. Liu, et 
al., 2008). Furthermore, inflammation and oxidative stress, both considered to be potential initiators and mediators of AF (J. Li, et al., 2010), and systemic concentrations of ADMA have been shown to be elevated in patients with persistent AF. In addition, ADMA levels fall after cardioversion (Goette, et al., 2012). In this context, ADMA levels and the L-Arg/ADMA ratio seem to be new biomarkers for the prediction of ventricular tachycardia/ventricular fibrillation episodes.

It is now demonstrated that PRMT proteins have tissue-specific functions and are regulated in a tissue-specific and age-dependent manner. PRMT1 expression was higher in the hearts of aged rats than in those of young rats (Hong, Lim, Lee, Oh, \& Kwon, 2012). The development of improved inhibitors, coupled with a better understanding of the cellular roles of PRMTs, is likely to demonstrate the feasibility of targeting PRMTs in specific immunological cells as a novel therapeutic strategy for immunomodulation during inflammation (Parry \& Ward, 2010). This hypothesis was supported by studies showing, for example, that pharmacological inhibition of DDAH results in an accumulation of ADMA and subsequently decreased NO formation (Rossiter, et al., 2005). At present, several L-Arg and $N^{5}$-(1-imino-alk(en)yl)-I-ornithine derivatives have been identified as DDAH inhibitors. These include $N^{5}$-(1-iminobut-3-enyl)-I-ornithine and $N^{\omega}$-(2-methoxyethyl)-L-Arg (Kotthaus, Schade, \& Clement, 2012; Schade, Kotthaus, \& Clement, 2010).

\subsection{Selective NADPH oxidase (NOX) inhibitors}

The molecular characteristics of the different NOX as therapeutic targets have been described previously in detail in intensive reviews. NOX inhibitors may block their electron transferring components, can remove and/or prevent assembly of the oxidase by interacting with their subunits at the molecular level. They may also reduce subunit gene and protein expression and modify the oxidases and/or their activators. A lot of substances have been screened to find a way to inhibit production of ROS, and thus protect the body from diseases (Jaquet, Scapozza, Clark, Krause, \& Lambeth, 2009; Schramm, Matusik, Osmenda, \& Guzik, 2012; Selemidis, Sobey, Wingler, Schmidt, \& Drummond, 2008).

\section{Natural NADPH oxidase inhibitors}

Recent studies have shown that berberine, a plant alkaloid, and emodin, an active component extracted from rhubarb, were able to inhibit NOX activity, reduce ROS and reduce gp91phox mRNA expression (Heo, Yun, Noh, \& Park, 2010; Sarna, Wu, Hwang, Siow, \& O, 2010). Similar effects were observed using treatment with 3-(4'-hydroxyl-3',5'-dimethoxyphenyl)propionic acid (HDMPPA), and ellagic acid, a polyphenol present in fruits (Lee, et al., 2010). The naturally-occurring methoxyphenol, apocynin, has been found to inhibit NOX upon activation by peroxidases (e.g. soybean peroxidase, myeloperoxidase) or ROS. Upon peroxidase-catalyzed activation, the apocynin oxidation products act to block the assembly and activation of NOX (Guzik \& Harrison, 2006). The ability of various polyphenols including apocynin to inhibit NOXs has been confirmed in a number of tissues including vessels. Moreover, new pharmacological actions of polyphenols, including their typical superoxide scavenging properties, have been investigated. In this field, the anti-inflammatory effect of apocynin has been demonstrated in various diseases. A recent study demonstrated that treatment with 
apocynin prevented tissue damage induced by splanchnic artery occlusion shock, and that this protection was associated with the blockage of NOX activity (Paterniti, et al., 2010). Apocynin has shown promising applications in animal studies of hypertension and other cardiovascular diseases (Yu, Weiwer, Linhardt, \& Dordick, 2008).

\section{NADPH oxidase chemical inhibitors}

Recently, pyrazolopyrimidine, tetrahydroindole and triazolopyrimidine (VAS 2870, VAS 3947) derivatives have been reported as specific NOX inhibitors that show promising potential as new drugs. Several other molecules are currently being studied and have been reviewed elsewhere. These include NOX inhibitors such as plumbagin, as well as polyphenolic derivative S17834 (J. A. Kim, et al., 2011). The major areas for the potential therapeutic applications of the compounds were ischemic heart disease, atherosclerosis, stroke and diabetic complications. One new approach concerns small interfering ribonucleic acids (siRNAs). These are short double stranded RNAs that inhibit, either partially or fully, the expression of mRNA, of its endogenous counterpart or of an exogenous gene. siRNAs selectively down-regulate NOX genes from NOX4, NOX1, NOX2, NOX5, for the potential treatment of various diseases related to NOX. There are an increasing number of reports about siRNA approaches directed against NOXs (Altenhofer, et al., 2012).The development of specific NOX inhibitors would greatly facilitate the elucidation of NOX function and would potentially provide clinically useful agents. At present, increasing attention is focused on the possibility that compounds that act specifically on ROS production by NOXs in specific areas such as the sarcoplasmic reticulum could serve as efficacious therapeutic agents (Sun, Hess, Wang, Miyagi, \& Stamler, 2012).

\section{Development of nitric oxide synthase inhibitors as therapeutic agents}

The over-production of $\mathrm{NO}$ is associated with many disorders including a wide range of functions in the neuronal (Parkinson's, Alzheimer's, Huntington's, headaches)(Alderton, Cooper, \& Knowles, 2001) immune, cardiovascular system (Naseem, 2005)and in a number of inflammatory diseases, for example, septic shock and rheumatoid arthritis. Inhibition of the isoforms of the enzyme should be a useful approach to treat these pathologies. Since the active sites of NOS are highly conserved, the virtual screening approach has been improved by taking into account the conformational plasticity of the target protein (Garcin, et al., 2008). Inhibitors of NOSs can be classified according to the site of inhibitor binding to the NOS. Four different classes of inhibitors have been recognized, the first of which interacts with the L-Arg -binding site.

\section{Substrate based inhibitors (Figure 6, A)}

Primarily, simple L-Arg derivatives were considered as inhibitors for experimental use because they were expected to compete with L- Arg for the active site of NOS. They serve as a perfect tool to inhibit NOS experimentally with a potential for clinical application (Vitecek, Lojek, Valacchi, \& Kubala, 2012)The best known inhibitors of NOS are amino acids related to the substrate L-Arg (Figure 6, A,2 and 3), for example: $\mathrm{N}^{G}$-monomethyl-L-Arg, (L-NMA) and L-N ${ }^{G}$-nitro L-Arg (L-NA) or its methyl ester LNAME. L-NMA is one of the first compounds to be intuitively employed to inhibit NOS. L-NMA has been used to determine the NO dependency of a physiological process. Since animal studies have 
revealed the possibility to attenuate the symptoms of septic shock with L-NMA, the use of this compound to treat patients with septic shock in clinical practice has attracted a great deal of attention (Lopez, et al., 2004). These compounds reversed the hypotension due to sepsis but did not increase survival.

Aminoguanidine selectively inhibits iNOS relative to eNOS but has many additional biological effects that are not related to NOS inhibition. Other inhibitors based on guanidines or isothioureas have been reported with various levels of selectivity and potency in vitro. Some inhibitors contain an amidine group, an analogue of the guanidinium moiety ofArg. Among these inhibitors, efforts have been made to design synthetic Arg-based dipeptide agents (Figure 6,A,3). A series of dipeptide amides containing Arg- $\mathrm{NO}_{2}$ were synthesized and screened for biological activity in vitro against the three isoforms of NOS. The order and the chirality of the amino acids in the dipeptide amides have profound effects on the inhibitory potency as well as on isoform selectivity. (Huang, Martasek, Roman, Masters, \& Silverman, 1999).

More recently, mutagenesis, biochemistry, crystallography and drug design were combined to elucidate the structural basis for the iNOS selectivity of some quinazoline and aminopyridine inhibitors (Garcin, et al., 2008). Quinazoline inhibitors, spirocyclic quinazoline inhibitors and aminopyridine inhibitors were more potent and more selective inhibitors of iNOS than eNOS and nNOS. Because nNOS is abundant in neuronal cells, but eNOS is vital in maintaining vascular tone in the brain, it became important to improve the inhibitory selectivity of nNOS over eNOS in order to lower the risk of side effects. A series of symmetric double-headed aminopyridines without charged groups were designed and recently synthesized (Xue, et al., 2011). However, the compounds exhibited low isoform selectivity. A series of small highly potent and selective nNOS inhibitors with a 2-aminopyridinomethyl pyrrolidine scaffold was reported (Huang, et al., 2013).. A series of transcyclopropyl- and methyl-containing nNOS inhibitors have been synthesized, and the impact of chirality at the cyclopropyl ring on inhibitor binding potency has been tested (H. Li, et al., 2013).

\section{Compounds interacting with tetrahydrobiopterin (Figure 6, B)}

The second class includes a set of compounds that interact with tetrahydrobiopterin. The importance of $\mathrm{BH}_{4}$ as a critical regulator of NOS function suggests that $\mathrm{BH}_{4}$ may be a rational therapeutic target in vascular disease states. Indeed, several studies have already explored the effect of $\mathrm{BH}_{4}$ administration on endothelial function (Fukuda, et al., 2002; Heitzer, et al., 2000). Given the limitations of direct $\mathrm{BH}_{4}$ administration, therapeutic interventions aimed at improving or preserving endogenous $\mathrm{BH}_{4}$ bioavailability may represent a viable alternative. For example, the beneficial effects of statins may depend in part on their ability to increase $\mathrm{BH}_{4}$ bioavailability in patients (Antoniades, et al., 2011). In contrast, the overproduction of NO by NOSs has been implicated in various pathological conditions including sepsis, inflammation, stroke, and neurodegenerative diseases. One an approach to target the $\mathrm{BH}_{4}$ binding site of NOS by appropriate inhibitors has been developed. The first generation of $\mathrm{BH}_{4}$-based NOS inhibitors was based on 4-amino $\mathrm{BH}_{4}$ derivatives (anti-pterins) in analogy to anti-folates such as methotrexate (Kotsonis, et al., 2001). In this field, a new approach had been to obtain a novel series of pterin-based NOS inhibitors based on an intact 4-oxo-pteridine 
nucleus. By systematically varying the substitution patterns in the 2-, 4-, 5-, 6-, and 7-positions of the 4-oxo-pteridine nucleus, these compounds were discovered to be effective NOS inhibitors (Matter \& Kotsonis, 2004).

\section{Inhibitors interacting with heme (Figure 6, C)}

The third class consists of inhibitors that interact directly with heme. One strategy to design inhibitors of heme enzymes is to attach an iron-binding moiety to a substrate backbone. This approach utilizes favorable contacts with the substrate binding pocket to fix the inhibitor in the active site. A crystal structure of a thioether-based inhibitor bound to nNOS was produced, and the crucial role of hydrophobic contacts in stabilizing thioether-heme coordination has been demonstrated (Martell, et al., 2010).

\section{Inhibitors interacting with calmodulin (CaM) cofactors}

The fourth class covers NOS inhibitors that interact with calmodulin (CaM) cofactors. Since eNOS and nNOS can reversibly bind $\mathrm{CaM}$ in a $\mathrm{Ca}^{2+}$-dependent manner, it has been demonstrated that CaM may be associated with nNOS as an inactive complex in cells at rest. In the linear sequence of eNOS, three CaM-binding fragments were identified: sequence 66-205, sequence 460-592, and sequence 505759. Synthetic peptides derived from these fragments were tested for their effects on CaM binding and eNOS catalytic activities. All the peptides significantly inhibit oxygenase activity in a concentration-dependent manner (Chen \& Wu, 2009).

\section{Summary and future directions}

Therefore, it appears that both a lack of and excess NO production in diseases can have various important pathological implications. Extensive clinical data support the notion that reducing ADMA levels may provide a novel therapeutic approach in the treatment of some cardiovascular diseases; reduced NO production being implicated in pathologies such as hypertension, atherosclerosis and ischemia/reperfusion. In the field of $\mathrm{BH}_{4}$, attention has shifted from structural and metabolicmolecular aspects to homoeostasis of the $\mathrm{BH}_{4}$ system and the pathophysiology of $\mathrm{BH}_{4}$ deficiency, with greater emphasis on the in vivo effects of NOS decoupling. The importance of $\mathrm{BH}_{4}$ as a critical regulator of eNOS function suggests that $\mathrm{BH}_{4}$ may be a rational therapeutic target in vascular disease states. NO at physiological levels may play an important role in the maintenance of vascular homeostasis while NO produced under oxidative stress conditions may evoke specific tissue injury. There is no doubt that NO is a janus-faced molecule and acts as a double-edged sword in cardiovascular injury. In addition, as inflammation, sepsis and stroke are associated with NO overproduction, the reduction of NO through the inhibition of NOS has the potential to be beneficial as an approach to develop new therapies for these diseases. While a number of NOS inhibitors were reported to have high affinity, the challenging task was to achieve high selectivity, each isoform being characterized by subcellular distribution, functional and catalytic properties. The application of modern inhibitor design approaches associated with a combination of mutagenesis should result in novel NOS modulators with enhanced isoform selectivity. 


\section{Conflict of interest statement}

The authors declare that they have no personal, financial or other relationships with other people or organizations within 3 years of beginning the work submitted that could inappropriately influence, or be perceived to influence, the work submitted.

\section{Acknowledgements}

The authors wish to thank Martine Goiset for secretarial assistance and Philip Bastable for English assistance.

This work was supported by grants from the French Ministry of Research, from the Institut National de la Santé et de la Recherche Médicale (INSERM) and from the Regional Council of Burgundy. 


\section{References}

Abbasi, F., Asagmi, T., Cooke, J. P., Lamendola, C., McLaughlin, T., Reaven, G. M., Stuehlinger, M., \& Tsao, P. S. (2001). Plasma concentrations of asymmetric dimethylarginine are increased in patients with type 2 diabetes mellitus. Am J Cardiol, 88, 1201-1203.

Abhary, S., Burdon, K. P., Kuot, A., Javadiyan, S., Whiting, M. J., Kasmeridis, N., Petrovsky, N., \& Craig, J. E. (2010). Sequence variation in DDAH1 and DDAH2 genes is strongly and additively associated with serum ADMA concentrations in individuals with type 2 diabetes. PLoS One, 5, e9462.

Alderton, W. K., Cooper, C. E., \& Knowles, R. G. (2001). Nitric oxide synthases: structure, function and inhibition. Biochemical Journal, 357, 593-615.

Alp, N. J., \& Channon, K. M. (2004). Regulation of endothelial nitric oxide synthase by tetrahydrobiopterin in vascular disease. Arterioscler Thromb Vasc Biol, 24, 413-420.

Altenhofer, S., Kleikers, P. W., Radermacher, K. A., Scheurer, P., Rob Hermans, J. J., Schiffers, P., Ho, H., Wingler, K., \& Schmidt, H. H. (2012). The NOX toolbox: validating the role of NADPH oxidases in physiology and disease. Cell Mol Life Sci, 69, 2327-2343.

Andersson, K., Gaudiot, N., Ribiere, C., Elizalde, M., Giudicelli, Y., \& Arner, P. (1999). A nitric oxidemediated mechanism regulates lipolysis in human adipose tissue in vivo. British Journal of Pharmacology, 126, 1639-1645.

Andreozzi, F., Presta, I., Mannino, G. C., Scarpelli, D., Di Silvestre, S., Di Pietro, N., Succurro, E., Sciacqua, A., Pandolfi, A., Consoli, A., Hribal, M. L., Perticone, F., \& Sesti, G. (2012). A functional variant of the dimethylarginine dimethylaminohydrolase-2 gene is associated with insulin sensitivity. PLoS One, 7, e36224.

Antoniades, C., Bakogiannis, C., Leeson, P., Guzik, T. J., Zhang, M. H., Tousoulis, D., Antonopoulos, A. S., Demosthenous, M., Marinou, K., Hale, A., Paschalis, A., Psarros, C., Triantafyllou, C., Bendall, J., Casadei, B., Stefanadis, C., \& Channon, K. M. (2011). Rapid, direct effects of statin treatment on arterial redox state and nitric oxide bioavailability in human atherosclerosis via tetrahydrobiopterin-mediated endothelial nitric oxide synthase coupling. Circulation, 124, 335-345.

Antoniades, C., Shirodaria, C., Leeson, P., Antonopoulos, A., Warrick, N., Van-Assche, T., Cunnington, C., Tousoulis, D., Pillai, R., Ratnatunga, C., Stefanadis, C., \& Channon, K. M. (2009). Association of plasma asymmetrical dimethylarginine (ADMA) with elevated vascular superoxide production and endothelial nitric oxide synthase uncoupling: implications for endothelial function in human atherosclerosis. Eur Heart J, 30, 1142-1150.

Antoniades, C., Shirodaria, C., Warrick, N., Cai, S., de Bono, J., Lee, J., Leeson, P., Neubauer, S., Ratnatunga, C., Pillai, R., Refsum, H., \& Channon, K. M. (2006). 5-methyltetrahydrofolate rapidly improves endothelial function and decreases superoxide production in human vessels: effects on vascular tetrahydrobiopterin availability and endothelial nitric oxide synthase coupling. Circulation, 114, 1193-1201.

Aoki, C., Nakano, A., Tanaka, S., Yanagi, K., Ohta, S., Jojima, T., Kasai, K., Takekawa, H., Hirata, K., \& Hattori, Y. (2012). Fluvastatin upregulates endothelial nitric oxide synthase activity via enhancement of its phosphorylation and expression and via an increase in tetrahydrobiopterin in vascular endothelial cells. Int J Cardiol, 156, 55-61.

Asagami, T., Abbasi, F., Stuelinger, M., Lamendola, C., McLaughlin, T., Cooke, J. P., Reaven, G. M., \& Tsao, P. S. (2002). Metformin treatment lowers asymmetric dimethylarginine concentrations in patients with type 2 diabetes. Metabolism, 51, 843-846.

Bedford, M. T., \& Clarke, S. G. (2009). Protein arginine methylation in mammals: who, what, and why. Mol Cell, 33, 1-13. 
Ben Baouali, A., Aube, H., Maupoil, V., Blettery, B., \& Rochette, L. (1994). Plasma lipid peroxidation in critically ill patients: importance of mechanical ventilation. Free Radical Biology \& Medicine, 16, 223-227.

Boger, R. H. (2003). Association of asymmetric dimethylarginine and endothelial dysfunction. Clin Chem Lab Med, 41, 1467-1472.

Boger, R. H., Maas, R., Schulze, F., \& Schwedhelm, E. (2009). Asymmetric dimethylarginine (ADMA) as a prospective marker of cardiovascular disease and mortality--an update on patient populations with a wide range of cardiovascular risk. Pharmacological Research, 60, 481-487.

Boger, R. H., Sullivan, L. M., Schwedhelm, E., Wang, T. J., Maas, R., Benjamin, E. J., Schulze, F., Xanthakis, V., Benndorf, R. A., \& Vasan, R. S. (2009). Plasma asymmetric dimethylarginine and incidence of cardiovascular disease and death in the community. Circulation, 119, 15921600.

Bray, G. A., Nielsen, S. J., \& Popkin, B. M. (2004). Consumption of high-fructose corn syrup in beverages may play a role in the epidemic of obesity. Am J Clin Nutr, 79, 537-543.

Brittes, J., Lucio, M., Nunes, C., Lima, J. L., \& Reis, S. (2010). Effects of resveratrol on membrane biophysical properties: relevance for its pharmacological effects. Chem Phys Lipids, 163, 747754.

Candipan, R. C., Wang, B. Y., Buitrago, R., Tsao, P. S., \& Cooke, J. P. (1996). Regression or progression. Dependency on vascular nitric oxide. Arterioscler Thromb Vasc Biol, 16, 44-50.

Capettini, L. S., Cortes, S. F., Silva, J. F., Alvarez-Leite, J. I., \& Lemos, V. S. (2011). Decreased production of neuronal NOS-derived hydrogen peroxide contributes to endothelial dysfunction in atherosclerosis. British Journal of Pharmacology, 164, 1738-1748.

Cardounel, A. J., Cui, H., Samouilov, A., Johnson, W., Kearns, P., Tsai, A. L., Berka, V., \& Zweier, J. L. (2007). Evidence for the pathophysiological role of endogenous methylarginines in regulation of endothelial NO production and vascular function. Journal of Biological Chemistry, 282, 879-887.

Cardounel, A. J., Xia, Y., \& Zweier, J. L. (2005). Endogenous methylarginines modulate superoxide as well as nitric oxide generation from neuronal nitric-oxide synthase: differences in the effects of monomethyl- and dimethylarginines in the presence and absence of tetrahydrobiopterin. Journal of Biological Chemistry, 280, 7540-7549.

Carlstrom, M., Persson, A. E., Larsson, E., Hezel, M., Scheffer, P. G., Teerlink, T., Weitzberg, E., \& Lundberg, J. O. (2011). Dietary nitrate attenuates oxidative stress, prevents cardiac and renal injuries, and reduces blood pressure in salt-induced hypertension. Cardiovascular Research, 89, 574-585.

Cha, B., \& Jho, E. H. (2012). Protein arginine methyltransferases (PRMTs) as therapeutic targets. Expert Opin Ther Targets, 16, 651-664.

Cheang, W. S., Wong, W. T., Tian, X. Y., Yang, Q., Lee, H. K., He, G. W., Yao, X., \& Huang, Y. (2011). Endothelial nitric oxide synthase enhancer reduces oxidative stress and restores endothelial function in db/db mice. Cardiovascular Research, 92, 267-275.

Chen, P. F., \& Wu, K. K. (2009). Two synthetic peptides corresponding to the proximal heme-binding domain and CD1 domain of human endothelial nitric-oxide synthase inhibit the oxygenase activity by interacting with CaM. Archives of Biochemistry \& Biophysics, 486, 132-140.

Cheng, D., Yadav, N., King, R. W., Swanson, M. S., Weinstein, E. J., \& Bedford, M. T. (2004). Small molecule regulators of protein arginine methyltransferases. Journal of Biological Chemistry, 279, 23892-23899.

Chin-Dusting, J. P., Willems, L., \& Kaye, D. M. (2007). L-arginine transporters in cardiovascular disease: a novel therapeutic target. Pharmacol Ther, 116, 428-436.

Clermont, G., Lecour, S., Vergely, C., Zeller, M., Perrin, C., Maupoil, V., Bouchot, O., \& Rochette, L. (2003). Direct demonstration of nitric oxide formation in organs of rabbits treated by transdermal glyceryl trinitrate using an in vivo spin trapping technique. Fundam Clin Pharmacol, 17, 709-715. 
Cottin, Y., Doise, J. M., Maupoil, V., Tanniere-Zeller, M., Dalloz, F., Maynadie, M., Walker, M. K., Louis, P., Carli, P. M., Wolf, J. E., \& Rochette, L. (1998). Plasma iron status and lipid peroxidation following thrombolytic therapy for acute myocardial infarction. Fundam Clin Pharmacol, 12, 236-241.

Crabtree, M. J., \& Channon, K. M. (2011). Synthesis and recycling of tetrahydrobiopterin in endothelial function and vascular disease. Nitric Oxide, 25, 81-88.

Crabtree, M. J., Smith, C. L., Lam, G., Goligorsky, M. S., \& Gross, S. S. (2008). Ratio of 5,6,7,8tetrahydrobiopterin to 7,8-dihydrobiopterin in endothelial cells determines glucose-elicited changes in NO vs. superoxide production by eNOS. Am J Physiol Heart Circ Physiol, 294, H1530-1540.

Daff, S. (2010). NO synthase: structures and mechanisms. Nitric Oxide, 23, 1-11.

Davids, M., van Hell, A. J., Visser, M., Nijveldt, R. J., van Leeuwen, P. A., \& Teerlink, T. (2012). Role of the human erythrocyte in generation and storage of asymmetric dimethylarginine. $A m \mathrm{~J}$ Physiol Heart Circ Physiol, 302, H1762-1770.

Delles, C., Schneider, M. P., John, S., Gekle, M., \& Schmieder, R. E. (2002). Angiotensin converting enzyme inhibition and angiotensin II AT1-receptor blockade reduce the levels of asymmetrical $N(G), N(G)$-dimethylarginine in human essential hypertension. Am J Hypertens, 15, 590-593.

Duell, P. B., \& Malinow, M. R. (1997). Homocyst(e)ine: an important risk factor for atherosclerotic vascular disease. Curr Opin Lipidol, 8, 28-34.

Ellger, B., Richir, M. C., van Leeuwen, P. A., Debaveye, Y., Langouche, L., Vanhorebeek, I., Teerlink, T., \& Van den Berghe, G. (2008). Glycemic control modulates arginine and asymmetricaldimethylarginine levels during critical illness by preserving dimethylargininedimethylaminohydrolase activity. Endocrinology, 149, 3148-3157.

Fadini, G. P., Albiero, M., Vigili de Kreutzenberg, S., Boscaro, E., Cappellari, R., Marescotti, M., Poncina, N., Agostini, C., \& Avogaro, A. (2012). Diabetes Impairs Stem Cell and Proangiogenic Cell Mobilization in Humans. Diabetes Care, In Press.

Feng, Y., Li, M., Wang, B., \& Zheng, Y. G. (2010). Discovery and mechanistic study of a class of protein arginine methylation inhibitors. J Med Chem, 53, 6028-6039.

Fink, B., Dikalov, S., \& Fink, N. (2006). ESR techniques for the detection of nitric oxide in vivo as an index of endothelial function. Pharmacol Rep, 58 Suppl, 8-15.

Forstermann, U., \& Sessa, W. C. (2012). Nitric oxide synthases: regulation and function. Eur Heart J, 33, 829-837, 837a-837d.

Fraccarollo, D., Widder, J. D., Galuppo, P., Thum, T., Tsikas, D., Hoffmann, M., Ruetten, H., Ertl, G., \& Bauersachs, J. (2008). Improvement in left ventricular remodeling by the endothelial nitric oxide synthase enhancer AVE9488 after experimental myocardial infarction. Circulation, 118, 818-827.

Frantz, S., Adamek, A., Fraccarollo, D., Tillmanns, J., Widder, J. D., Dienesch, C., Schafer, A., Podolskaya, A., Held, M., Ruetten, H., Ertl, G., \& Bauersachs, J. (2009). The eNOS enhancer AVE 9488: a novel cardioprotectant against ischemia reperfusion injury. Basic Res Cardiol, 104, 773-779.

Fukuda, Y., Teragawa, H., Matsuda, K., Yamagata, T., Matsuura, H., \& Chayama, K. (2002). Tetrahydrobiopterin restores endothelial function of coronary arteries in patients with hypercholesterolaemia. Heart, 87, 264-269.

Garcin, E. D., Arvai, A. S., Rosenfeld, R. J., Kroeger, M. D., Crane, B. R., Andersson, G., Andrews, G., Hamley, P. J., Mallinder, P. R., Nicholls, D. J., St-Gallay, S. A., Tinker, A. C., Gensmantel, N. P., Mete, A., Cheshire, D. R., Connolly, S., Stuehr, D. J., Aberg, A., Wallace, A. V., Tainer, J. A., \& Getzoff, E. D. (2008). Anchored plasticity opens doors for selective inhibitor design in nitric oxide synthase. Nat Chem Biol, 4, 700-707.

Gielis, J. F., Lin, J. Y., Wingler, K., Van Schil, P. E., Schmidt, H. H., \& Moens, A. L. (2011). Pathogenetic role of eNOS uncoupling in cardiopulmonary disorders. Free Radical Biology \& Medicine, 50, 765-776. 
Goette, A., Hammwohner, M., Bukowska, A., Scalera, F., Martens-Lobenhoffer, J., Dobrev, D., Ravens, U., Weinert, S., Medunjanin, S., Lendeckel, U., \& Bode-Boger, S. M. (2012). The impact of rapid atrial pacing on ADMA and endothelial NOS. Int J Cardiol, 154, 141-146.

Gomes, E., Duarte, R., Reis, R. P., Candido, A., Cardim, N., Correia, M. J., Castela, S., Cordeiro, R., Ramos, A., Lobo, J. L., \& Correia, J. F. (2002). Homocysteine increase after acute myocardial infarction--can it explain the differences between case-control and cohort studies? Rev Port Cardiol, 21, 575-581.

Goonasekera, C. D., Rees, D. D., Woolard, P., Frend, A., Shah, V., \& Dillon, M. J. (1997). Nitric oxide synthase inhibitors and hypertension in children and adolescents. J Hypertens, 15, 901-909.

Gruber, J., Schaffer, S., \& Halliwell, B. (2008). The mitochondrial free radical theory of ageing--where do we stand? Front Biosci, 13, 6554-6579.

Guzik, T. J., \& Harrison, D. G. (2006). Vascular NADPH oxidases as drug targets for novel antioxidant strategies. Drug Discov Today, 11, 524-533.

Hagensen, M. K., Vanhoutte, P. M., \& Bentzon, J. F. (2012). Arterial endothelial cells: still the craftsmen of regenerated endothelium. Cardiovascular Research, 95, 281-289.

Halliwell, B., \& Gutteridge, J. M. (1995). The definition and measurement of antioxidants in biological systems. Free Radical Biology \& Medicine, 18, 125-126.

Hamed, S., Brenner, B., \& Roguin, A. (2011). Nitric oxide: a key factor behind the dysfunctionality of endothelial progenitor cells in diabetes mellitus type-2. Cardiovascular Research, 91, 9-15.

Heinke, R., Spannhoff, A., Meier, R., Trojer, P., Bauer, I., Jung, M., \& Sippl, W. (2009). Virtual screening and biological characterization of novel histone arginine methyltransferase PRMT1 inhibitors. ChemMedChem, 4, 69-77.

Heitzer, T., Krohn, K., Albers, S., \& Meinertz, T. (2000). Tetrahydrobiopterin improves endotheliumdependent vasodilation by increasing nitric oxide activity in patients with Type II diabetes mellitus. Diabetologia, 43, 1435-1438.

Heo, S. K., Yun, H. J., Noh, E. K., \& Park, S. D. (2010). Emodin and rhein inhibit LIGHT-induced monocytes migration by blocking of ROS production. Vascul Pharmacol, 53, 28-37.

Hingorani, A. D., Liang, C. F., Fatibene, J., Lyon, A., Monteith, S., Parsons, A., Haydock, S., Hopper, R. V., Stephens, N. G., O'Shaughnessy, K. M., \& Brown, M. J. (1999). A common variant of the endothelial nitric oxide synthase (Glu298-->Asp) is a major risk factor for coronary artery disease in the UK. Circulation, 100, 1515-1520.

Holden, D. P., Cartwright, J. E., Nussey, S. S., \& Whitley, G. S. (2003). Estrogen stimulates dimethylarginine dimethylaminohydrolase activity and the metabolism of asymmetric dimethylarginine. Circulation, 108, 1575-1580.

Hong, E., Lim, Y., Lee, E., Oh, M., \& Kwon, D. (2012). Tissue-specific and age-dependent expression of protein arginine methyltransferases (PRMTs) in male rat tissues. Biogerontology, 13, 329336.

Huang, H., Li, H., Martasek, P., Roman, L. J., Poulos, T. L., \& Silverman, R. B. (2013). Structure-Guided Design of Selective Inhibitors of Neuronal Nitric Oxide Synthase. J Med Chem.

Huang, H., Martasek, P., Roman, L. J., Masters, B. S., \& Silverman, R. B. (1999). N(omega)Nitroarginine-containing dipeptide amides. Potent and highly selective inhibitors of neuronal nitric oxide synthase. J Med Chem, 42, 3147-3153.

Imanishi, T., Tsujioka, H., Ikejima, H., Kuroi, A., Takarada, S., Kitabata, H., Tanimoto, T., Muragaki, Y., Mochizuki, S., Goto, M., Yoshida, K., \& Akasaka, T. (2008). Renin inhibitor aliskiren improves impaired nitric oxide bioavailability and protects against atherosclerotic changes. Hypertension, 52, 563-572.

Jacobi, J., Maas, R., Cordasic, N., Koch, K., Schmieder, R. E., Boger, R. H., \& Hilgers, K. F. (2008). Role of asymmetric dimethylarginine for angiotensin II-induced target organ damage in mice. Am J Physiol Heart Circ Physiol, 294, H1058-1066.

Jaquet, V., Scapozza, L., Clark, R. A., Krause, K. H., \& Lambeth, J. D. (2009). Small-molecule NOX inhibitors: ROS-generating NADPH oxidases as therapeutic targets. Antioxid Redox Signal, 11, 2535-2552. 
Jones, D. P. (2006). Redefining oxidative stress. Antioxid Redox Signal, 8, 1865-1879.

Jones, D. P., \& Go, Y. M. (2010). Redox compartmentalization and cellular stress. Diabetes Obes Metab, 12 Suppl 2, 116-125.

Kannel, W. B., Feinleib, M., McNamara, P. M., Garrison, R. J., \& Castelli, W. P. (1979). An investigation of coronary heart disease in families. The Framingham offspring study. Am J Epidemiol, 110, 281-290.

Katusic, Z. S., d'Uscio, L. V., \& Nath, K. A. (2009). Vascular protection by tetrahydrobiopterin: progress and therapeutic prospects. Trends Pharmacol Sci, 30, 48-54.

Kawata, T., Daimon, M., Hasegawa, R., Teramoto, K., Toyoda, T., Sekine, T., Yamamoto, K., Uchida, D., Himi, T., Yoshida, K., \& Komuro, I. (2009). Effect of angiotensin-converting enzyme inhibitor on serum asymmetric dimethylarginine and coronary circulation in patients with type 2 diabetes mellitus. Int J Cardiol, 132, 286-288.

Kielstein, J. T., Bode-Boger, S. M., Frolich, J. C., Ritz, E., Haller, H., \& Fliser, D. (2003). Asymmetric dimethylarginine, blood pressure, and renal perfusion in elderly subjects. Circulation, 107, 1891-1895.

Kim, H. L., \& Park, Y. S. (2010). Maintenance of cellular tetrahydrobiopterin homeostasis. BMB Rep, $43,584-592$.

Kim, J. A., Neupane, G. P., Lee, E. S., Jeong, B. S., Park, B. C., \& Thapa, P. (2011). NADPH oxidase inhibitors: a patent review. Expert Opin Ther Pat, 21, 1147-1158.

Korandji, C., Zeller, M., Guilland, J. C., Collin, B., Lauzier, B., Sicard, P., Duvillard, L., Goirand, F., Moreau, D., Cottin, Y., Rochette, L., \& Vergely, C. (2011). Time course of asymmetric dimethylarginine (ADMA) and oxidative stress in fructose-hypertensive rats: a model related to metabolic syndrome. Atherosclerosis, 214, 310-315.

Korandji, C., Zeller, M., Guilland, J. C., Vergely, C., Sicard, P., Duvillard, L., Gambert, P., Moreau, D., Cottin, Y., \& Rochette, L. (2007). Asymmetric dimethylarginine (ADMA) and hyperhomocysteinemia in patients with acute myocardial infarction. Clin Biochem, 40, 66-72.

Kotsonis, P., Frohlich, L. G., Raman, C. S., Li, H., Berg, M., Gerwig, R., Groehn, V., Kang, Y., Al-Masoudi, N., Taghavi-Moghadam, S., Mohr, D., Munch, U., Schnabel, J., Martasek, P., Masters, B. S., Strobel, H., Poulos, T., Matter, H., Pfleiderer, W., \& Schmidt, H. H. (2001). Structural basis for pterin antagonism in nitric-oxide synthase. Development of novel 4-oxo-pteridine antagonists of (6R)-5,6,7,8-tetrahydrobiopterin. Journal of Biological Chemistry, 276, 4913349141.

Kotthaus, J., Schade, D., \& Clement, B. (2012). Designing modulators of dimethylarginine dimethylaminohydrolase (DDAH): a focus on selectivity over arginase. J Enzyme Inhib Med Chem, 27, 24-28.

Krause, C. D., Yang, Z. H., Kim, Y. S., Lee, J. H., Cook, J. R., \& Pestka, S. (2007). Protein arginine methyltransferases: evolution and assessment of their pharmacological and therapeutic potential. Pharmacol Ther, 113, 50-87.

Kuliszewski, M. A., Ward, M. R., Kowalewski, J. W., Smith, A. H., Stewart, D. J., Kutryk, M. J., \& LeongPoi, H. (2012). A direct comparison of endothelial progenitor cell dysfunction in rat metabolic syndrome and diabetes. Atherosclerosis, In Press.

Kuzkaya, N., Weissmann, N., Harrison, D. G., \& Dikalov, S. (2003). Interactions of peroxynitrite, tetrahydrobiopterin, ascorbic acid, and thiols: implications for uncoupling endothelial nitricoxide synthase. Journal of Biological Chemistry, 278, 22546-22554.

Lecour, S., Maupoil, V., Siri, O., Tabard, A., \& Rochette, L. (1999). Electron spin resonance detection of nitric oxide generation in major organs from LPS-treated rats. J Cardiovasc Pharmacol, 33, 78-85.

Lee, W. J., Ou, H. C., Hsu, W. C., Chou, M. M., Tseng, J. J., Hsu, S. L., Tsai, K. L., \& Sheu, W. H. (2010). Ellagic acid inhibits oxidized LDL-mediated LOX-1 expression, ROS generation, and inflammation in human endothelial cells. J Vasc Surg, 52, 1290-1300.

Leiper, J., \& Nandi, M. (2011). The therapeutic potential of targeting endogenous inhibitors of nitric oxide synthesis. Nat Rev Drug Discov, 10, 277-291. 
Li, H., Xue, F., Kraus, J. M., 2nd, Ji, H., Labby, K. J., Mataka, J., Delker, S. L., Martasek, P., Roman, L. J., Poulos, T. L., \& Silverman, R. B. (2013). Cyclopropyl- and methyl-containing inhibitors of neuronal nitric oxide synthase. Bioorg Med Chem, 21, 1333-1343.

Li, J., Solus, J., Chen, Q., Rho, Y. H., Milne, G., Stein, C. M., \& Darbar, D. (2010). Role of inflammation and oxidative stress in atrial fibrillation. Heart Rhythm, 7, 438-444.

Li, X. P., Zhou, Y., Zhao, S. P., Gao, M., Zhou, Q. C., \& Li, Y. S. (2004). Effect of endogenous estrogen on endothelial function in women with coronary heart disease and its mechanism. Clin Chim Acta, 339, 183-188.

Libby, P. (2008). The molecular mechanisms of the thrombotic complications of atherosclerosis. $J$ Intern Med, 263, 517-527.

Lin, K. Y., Ito, A., Asagami, T., Tsao, P. S., Adimoolam, S., Kimoto, M., Tsuji, H., Reaven, G. M., \& Cooke, J. P. (2002). Impaired nitric oxide synthase pathway in diabetes mellitus: role of asymmetric dimethylarginine and dimethylarginine dimethylaminohydrolase. Circulation, 106, 987-992.

Linz, W., Wohlfart, P., Baader, M., Breitschopf, K., Falk, E., Schafer, H. L., Gerl, M., Kramer, W., \& Rutten, H. (2009). The peroxisome proliferator-activated receptor-alpha (PPAR-alpha) agonist, AVE8134, attenuates the progression of heart failure and increases survival in rats. Acta Pharmacol Sin, 30, 935-946.

Liu, F., Zhao, X., Perna, F., Wang, L., Koppikar, P., Abdel-Wahab, O., Harr, M. W., Levine, R. L., Xu, H., Tefferi, A., Deblasio, A., Hatlen, M., Menendez, S., \& Nimer, S. D. (2011). JAK2V617Fmediated phosphorylation of PRMT5 downregulates its methyltransferase activity and promotes myeloproliferation. Cancer Cell, 19, 283-294.

Liu, H., Qu, X., Liang, Z., Chen, W., Xia, W., \& Song, Y. (2008). Variance of DDAH/PRMT/ADMA pathway in atrial fibrillation dogs. Biochem Biophys Res Commun, 377, 884-888.

Lonn, E., Yusuf, S., Arnold, M. J., Sheridan, P., Pogue, J., Micks, M., McQueen, M. J., Probstfield, J., Fodor, G., Held, C., \& Genest, J., Jr. (2006). Homocysteine lowering with folic acid and B vitamins in vascular disease. N Engl J Med, 354, 1567-1577.

Lopez, A., Lorente, J. A., Steingrub, J., Bakker, J., McLuckie, A., Willatts, S., Brockway, M., Anzueto, A., Holzapfel, L., Breen, D., Silverman, M. S., Takala, J., Donaldson, J., Arneson, C., Grove, G., Grossman, S., \& Grover, R. (2004). Multiple-center, randomized, placebo-controlled, doubleblind study of the nitric oxide synthase inhibitor 546C88: effect on survival in patients with septic shock. Crit Care Med, 32, 21-30.

Lorgis, L., Zeller, M., Dentan, G., Sicard, P., Richard, C., Buffet, P., L'Huillier, I., Beer, J. C., Cottin, Y., Rochette, L., \& Vergely, C. (2010). The free oxygen radicals test (FORT) to assess circulating oxidative stress in patients with acute myocardial infarction. Atherosclerosis, 213, 616-621.

Luo, Z., Teerlink, T., Griendling, K., Aslam, S., Welch, W. J., \& Wilcox, C. S. (2010). Angiotensin II and NADPH oxidase increase ADMA in vascular smooth muscle cells. Hypertension, 56, 498-504.

Luther, J. M., \& Brown, N. J. (2011). The renin-angiotensin-aldosterone system and glucose homeostasis. Trends Pharmacol Sci, 32, 734-739.

Marcovecchio, M. L., Widmer, B., Dunger, D. B., \& Dalton, R. N. (2008). Effect of acute variations of insulin and glucose on plasma concentrations of asymmetric dimethylarginine in young people with Type 1 diabetes. Clin Sci (Lond), 115, 361-369.

Martell, J. D., Li, H., Doukov, T., Martasek, P., Roman, L. J., Soltis, M., Poulos, T. L., \& Silverman, R. B. (2010). Heme-coordinating inhibitors of neuronal nitric oxide synthase. Iron-thioether coordination is stabilized by hydrophobic contacts without increased inhibitor potency. J Am Chem Soc, 132, 798-806.

Martin, C., Dubouchaud, H., Mosoni, L., Chardigny, J. M., Oudot, A., Fontaine, E., Vergely, C., Keriel, C., Rochette, L., Leverve, X., \& Demaison, L. (2007). Abnormalities of mitochondrial functioning can partly explain the metabolic disorders encountered in sarcopenic gastrocnemius. Aging Cell, 6, 165-177.

Martinez-Gonzalez, J., Raposo, B., Rodriguez, C., \& Badimon, L. (2001). 3-hydroxy-3-methylglutaryl coenzyme a reductase inhibition prevents endothelial NO synthase downregulation by 
atherogenic levels of native LDLs: balance between transcriptional and posttranscriptional regulation. Arterioscler Thromb Vasc Biol, 21, 804-809.

Masuda, H., Goto, M., Tamaoki, S., \& Azuma, H. (1999). Accelerated intimal hyperplasia and increased endogenous inhibitors for NO synthesis in rabbits with alloxan-induced hyperglycaemia. British Journal of Pharmacology, 126, 211-218.

Matetzky, S., Freimark, D., Ben-Ami, S., Goldenberg, I., Leor, J., Doolman, R., Novikov, I., Eldar, M., \& Hod, H. (2003). Association of elevated homocysteine levels with a higher risk of recurrent coronary events and mortality in patients with acute myocardial infarction. Arch Intern Med, 163, 1933-1937.

Matter, H., \& Kotsonis, P. (2004). Biology and chemistry of the inhibition of nitric oxide synthases by pteridine-derivatives as therapeutic agents. Med Res Rev, 24, 662-684.

Michel, T., \& Vanhoutte, P. M. (2010). Cellular signaling and NO production. Pflugers Arch, 459, 807816.

Miura, T., Muraoka, S., Ikeda, N., Watanabe, M., \& Fujimoto, Y. (2000). Antioxidative and prooxidative action of stilbene derivatives. Pharmacol Toxicol, 86, 203-208.

Miyazaki, H., Matsuoka, H., Cooke, J. P., Usui, M., Ueda, S., Okuda, S., \& Imaizumi, T. (1999). Endogenous nitric oxide synthase inhibitor: a novel marker of atherosclerosis. Circulation, 99, 1141-1146.

Moens, A. L., Ketner, E. A., Takimoto, E., Schmidt, T. S., O'Neill, C. A., Wolin, M. S., Alp, N. J., Channon, K. M., \& Kass, D. A. (2011). Bi-modal dose-dependent cardiac response to tetrahydrobiopterin in pressure-overload induced hypertrophy and heart failure. Journal of Molecular \& Cellular Cardiology, 51, 564-569.

Moens, A. L., Takimoto, E., Tocchetti, C. G., Chakir, K., Bedja, D., Cormaci, G., Ketner, E. A., Majmudar, M., Gabrielson, K., Halushka, M. K., Mitchell, J. B., Biswal, S., Channon, K. M., Wolin, M. S., Alp, N. J., Paolocci, N., Champion, H. C., \& Kass, D. A. (2008). Reversal of cardiac hypertrophy and fibrosis from pressure overload by tetrahydrobiopterin: efficacy of recoupling nitric oxide synthase as a therapeutic strategy. Circulation, 117, 2626-2636.

Montezano, A. C., \& Touyz, R. M. (2012). Reactive oxygen species and endothelial function--role of nitric oxide synthase uncoupling and Nox family nicotinamide adenine dinucleotide phosphate oxidases. Basic Clin Pharmacol Toxicol, 110, 87-94.

Mori, E., Haramaki, N., Ikeda, H., \& Imaizumi, T. (1998). Intra-coronary administration of L-arginine aggravates myocardial stunning through production of peroxynitrite in dogs. Cardiovascular Research, 40, 113-123.

Naseem, K. M. (2005). The role of nitric oxide in cardiovascular diseases. Mol Aspects Med, 26, 33-65.

Nunes, S. F., Figueiredo, I. V., Pereira, J. S., de Lemos, E. T., Reis, F., Teixeira, F., \& Caramona, M. M. (2011). Monoamine oxidase and semicarbazide-sensitive amine oxidase kinetic analysis in mesenteric arteries of patients with type 2 diabetes. Physiol Res, 60, 309-315.

Onozato, M. L., Tojo, A., Leiper, J., Fujita, T., Palm, F., \& Wilcox, C. S. (2008). Expression of NG,NGdimethylarginine dimethylaminohydrolase and protein arginine $\mathrm{N}$-methyltransferase isoforms in diabetic rat kidney: effects of angiotensin II receptor blockers. Diabetes, 57, 172180.

Oron-Herman, M., Kamari, Y., Grossman, E., Yeger, G., Peleg, E., Shabtay, Z., Shamiss, A., \& Sharabi, Y. (2008). Metabolic syndrome: comparison of the two commonly used animal models. Am J Hypertens, 21, 1018-1022.

Oudot, A., Martin, C., Busseuil, D., Vergely, C., Demaison, L., \& Rochette, L. (2006). NADPH oxidases are in part responsible for increased cardiovascular superoxide production during aging. Free Radical Biology \& Medicine, 40, 2214-2222.

Pabla, R., \& Curtis, M. J. (2007). Nitric oxide fails to confer endogenous antiarrhythmic cardioprotection in the primate heart in vitro. British Journal of Pharmacology, 150, 893-898.

Padilla, F., Garcia-Dorado, D., Agullo, L., Inserte, J., Paniagua, A., Mirabet, S., Barrabes, J. A., RuizMeana, M., \& Soler-Soler, J. (2000). L-Arginine administration prevents reperfusion-induced 
cardiomyocyte hypercontracture and reduces infarct size in the pig. Cardiovascular Research, 46, 412-420.

Paiva, H., Lehtimaki, T., Laakso, J., Ruokonen, I., Rantalaiho, V., Wirta, O., Pasternack, A., \& Laaksonen, R. (2003). Plasma concentrations of asymmetric-dimethyl-arginine in type 2 diabetes associate with glycemic control and glomerular filtration rate but not with risk factors of vasculopathy. Metabolism, 52, 303-307.

Pal, S., Baiocchi, R. A., Byrd, J. C., Grever, M. R., Jacob, S. T., \& Sif, S. (2007). Low levels of miR-92b/96 induce PRMT5 translation and H3R8/H4R3 methylation in mantle cell lymphoma. EMBO J, 26, 3558-3569.

Palm, F., Onozato, M. L., Luo, Z., \& Wilcox, C. S. (2007). Dimethylarginine dimethylaminohydrolase (DDAH): expression, regulation, and function in the cardiovascular and renal systems. Am J Physiol Heart Circ Physiol, 293, H3227-3245.

Palomo, I., Contreras, A., Alarcon, L. M., Leiva, E., Guzman, L., Mujica, V., Icaza, G., Diaz, N., Gonzalez, D. R., \& Moore-Carrasco, R. (2011). Elevated concentration of asymmetric dimethylarginine (ADMA) in individuals with metabolic syndrome. Nitric Oxide, 24, 224-228.

Parry, R. V., \& Ward, S. G. (2010). Protein arginine methylation: a new handle on T lymphocytes? Trends Immunol, 31, 164-169.

Pasini, A. F., Garbin, U., Stranieri, C., Boccioletti, V., Mozzini, C., Manfro, S., Pasini, A., Cominacini, M., \& Cominacini, L. (2008). Nebivolol treatment reduces serum levels of asymmetric dimethylarginine and improves endothelial dysfunction in essential hypertensive patients. Am J Hypertens, 21, 1251-1257.

Paterniti, I., Galuppo, M., Mazzon, E., Impellizzeri, D., Esposito, E., Bramanti, P., \& Cuzzocrea, S. (2010). Protective effects of apocynin, an inhibitor of NADPH oxidase activity, in splanchnic artery occlusion and reperfusion. J Leukoc Biol, 88, 993-1003.

$\mathrm{Pi}, \mathrm{X}$. , \& Patterson, C. (2008). Twin layers of lightning: a new role for the chaperone Hsp90 in angiogenesis. Arterioscler Thromb Vasc Biol, 28, 6-7.

Pope, A. J., Karuppiah, K., \& Cardounel, A. J. (2009). Role of the PRMT-DDAH-ADMA axis in the regulation of endothelial nitric oxide production. Pharmacological Research, 60, 461-465.

Puntarulo, S. (2005). Iron, oxidative stress and human health. Mol Aspects Med, 26, 299-312.

Reaven, G. M. (2003). Insulin resistance/compensatory hyperinsulinemia, essential hypertension, and cardiovascular disease. J Clin Endocrinol Metab, 88, 2399-2403.

Rees, D. D., Palmer, R. M., Schulz, R., Hodson, H. F., \& Moncada, S. (1990). Characterization of three inhibitors of endothelial nitric oxide synthase in vitro and in vivo. British Journal of Pharmacology, 101, 746-752.

Richard, C., Lauzier, B., Delemasure, S., Talbot, S., Ghibu, S., Collin, B., Senecal, J., Menetrier, F., Vergely, C., Couture, R., \& Rochette, L. (2008). Effects of angiotensin-1 converting enzyme inhibition on oxidative stress and bradykinin receptor expression during doxorubicin-induced cardiomyopathy in rats. J Cardiovasc Pharmacol, 52, 278-285.

Rochette, L., Cottin, Y., Zeller, M., \& Vergely, C. (2013). Carbon monoxide: mechanisms of action and potential clinical implications. Pharmacol Ther, 137, 133-152.

Rochette, L., Tatou, E., Maupoil, V., Zeller, M., Cottin, Y., Jazayeri, S., Brenot, R., Girard, C., David, M., \& Vergely, C. (2011). Atrial and vascular oxidative stress in patients with heart failure. Cell Physiol Biochem, 27, 497-502.

Rochette, L., \& Vergely, C. (2008). Forgotten radicals in biology. International Journal of Biomedical Sciences, 4, 255-259.

Roe, N. D., \& Ren, J. (2012). Nitric oxide synthase uncoupling: a therapeutic target in cardiovascular diseases. Vascul Pharmacol, 57, 168-172.

Roede, J. R., Go, Y. M., \& Jones, D. P. (2012). Redox equivalents and mitochondrial bioenergetics. Methods Mol Biol, 810, 249-280.

Roman, L. J., \& Masters, B. S. (2006). Electron transfer by neuronal nitric-oxide synthase is regulated by concerted interaction of calmodulin and two intrinsic regulatory elements. Journal of Biological Chemistry, 281, 23111-23118. 
Rossiter, S., Smith, C. L., Malaki, M., Nandi, M., Gill, H., Leiper, J. M., Vallance, P., \& Selwood, D. L. (2005). Selective substrate-based inhibitors of mammalian dimethylarginine dimethylaminohydrolase. J Med Chem, 48, 4670-4678.

Saliques, S., Teyssier, J. R., Vergely, C., Lorgis, L., Lorin, J., Farnier, M., Donzel, A., Sicard, P., Berchoud, J., Lagrost, A. C., Touzery, C., Ragot, S., Cottin, Y., Rochette, L., \& Zeller, M. (2011). Circulating leukocyte telomere length and oxidative stress: a new target for statin therapy. Atherosclerosis, 219, 753-760.

Sarna, L. K., Wu, N., Hwang, S. Y., Siow, Y. L., \& O, K. (2010). Berberine inhibits NADPH oxidase mediated superoxide anion production in macrophages. Can J Physiol Pharmacol, 88, 369378.

Sartori, C., \& Scherrer, U. (1999). Insulin, nitric oxide and the sympathetic nervous system: at the crossroads of metabolic and cardiovascular regulation. J Hypertens, 17, 1517-1525.

Sartorio, C. L., Fraccarollo, D., Galuppo, P., Leutke, M., Ertl, G., Stefanon, I., \& Bauersachs, J. (2007). Mineralocorticoid receptor blockade improves vasomotor dysfunction and vascular oxidative stress early after myocardial infarction. Hypertension, 50, 919-925.

Sato, Y., Ito, T., Udaka, N., Kanisawa, M., Noguchi, Y., Cushman, S. W., \& Satoh, S. (1996). Immunohistochemical localization of facilitated-diffusion glucose transporters in rat pancreatic islets. Tissue Cell, 28, 637-643.

Satoh, M., Fujimoto, S., Arakawa, S., Yada, T., Namikoshi, T., Haruna, Y., Horike, H., Sasaki, T., \& Kashihara, N. (2008). Angiotensin II type 1 receptor blocker ameliorates uncoupled endothelial nitric oxide synthase in rats with experimental diabetic nephropathy. Nephrol Dial Transplant, 23, 3806-3813.

Satoh, M., Fujimoto, S., Haruna, Y., Arakawa, S., Horike, H., Komai, N., Sasaki, T., Tsujioka, K., Makino, H., \& Kashihara, N. (2005). NAD(P)H oxidase and uncoupled nitric oxide synthase are major sources of glomerular superoxide in rats with experimental diabetic nephropathy. Am J Physiol Renal Physiol, 288, F1144-1152.

Schade, D., Kotthaus, J., \& Clement, B. (2010). Modulating the NO generating system from a medicinal chemistry perspective: current trends and therapeutic options in cardiovascular disease. Pharmacol Ther, 126, 279-300.

Schmidt, K., Neubauer, A., Kolesnik, B., Stasch, J. P., Werner, E. R., Gorren, A. C., \& Mayer, B. (2012). Tetrahydrobiopterin protects soluble guanylate cyclase against oxidative inactivation. $\mathrm{Mol}$ Pharmacol, 82, 420-427.

Schnabel, R., Blankenberg, S., Lubos, E., Lackner, K. J., Rupprecht, H. J., Espinola-Klein, C., Jachmann, N., Post, F., Peetz, D., Bickel, C., Cambien, F., Tiret, L., \& Munzel, T. (2005). Asymmetric dimethylarginine and the risk of cardiovascular events and death in patients with coronary artery disease: results from the AtheroGene Study. Circulation Research, 97, e53-59.

Schramm, A., Matusik, P., Osmenda, G., \& Guzik, T. J. (2012). Targeting NADPH oxidases in vascular pharmacology. Vascul Pharmacol, 56, 216-231.

Schulman, S. P., Becker, L. C., Kass, D. A., Champion, H. C., Terrin, M. L., Forman, S., Ernst, K. V., Kelemen, M. D., Townsend, S. N., Capriotti, A., Hare, J. M., \& Gerstenblith, G. (2006). Larginine therapy in acute myocardial infarction: the Vascular Interaction With Age in Myocardial Infarction (VINTAGE MI) randomized clinical trial. Jama, 295, 58-64.

Schulz, E., Jansen, T., Wenzel, P., Daiber, A., \& Munzel, T. (2008). Nitric oxide, tetrahydrobiopterin, oxidative stress, and endothelial dysfunction in hypertension. Antioxid Redox Signal, 10, $1115-1126$.

Searles, C. D. (2002). The nitric oxide pathway and oxidative stress in heart failure. Congest Heart Fail, 8, 142-147, 155.

Selemidis, S., Sobey, C. G., Wingler, K., Schmidt, H. H., \& Drummond, G. R. (2008). NADPH oxidases in the vasculature: molecular features, roles in disease and pharmacological inhibition. Pharmacol Ther, 120, 254-291. 
Shimizu, S., Hiroi, T., Ishii, M., Hagiwara, T., Wajima, T., Miyazaki, A., \& Kiuchi, Y. (2008). Hydrogen peroxide stimulates tetrahydrobiopterin synthesis through activation of the Jak2 tyrosine kinase pathway in vascular endothelial cells. Int J Biochem Cell Biol, 40, 755-765.

Sicard, P., Delemasure, S., Korandji, C., Segueira-Le Grand, A., Lauzier, B., Guilland, J. C., Duvillard, L., Zeller, M., Cottin, Y., Vergely, C., \& Rochette, L. (2008). Anti-hypertensive effects of Rosuvastatin are associated with decreased inflammation and oxidative stress markers in hypertensive rats. Free Radical Research, 42, 226-236.

Siroen, M. P., Teerlink, T., Nijveldt, R. J., Prins, H. A., Richir, M. C., \& van Leeuwen, P. A. (2006). The clinical significance of asymmetric dimethylarginine. Annu Rev Nutr, 26, 203-228.

Stuhlinger, M. C., Tsao, P. S., Her, J. H., Kimoto, M., Balint, R. F., \& Cooke, J. P. (2001). Homocysteine impairs the nitric oxide synthase pathway: role of asymmetric dimethylarginine. Circulation, 104, 2569-2575.

Sun, Q. A., Hess, D. T., Wang, B., Miyagi, M., \& Stamler, J. S. (2012). Off-target thiol alkylation by the NADPH oxidase inhibitor 3-benzyl-7-(2-benzoxazolyl)thio-1,2,3-triazolo[4,5-d]pyrimidine (VAS2870). Free Radical Biology \& Medicine, 52, 1897-1902.

Svajger, U., \& Jeras, M. (2012). Anti-inflammatory effects of resveratrol and its potential use in therapy of immune-mediated diseases. Int Rev Immunol, 31, 202-222.

Teerlink, T. (2005). ADMA metabolism and clearance. Vasc Med, 10 Suppl 1, S73-81.

Teerlink, T., Luo, Z., Palm, F., \& Wilcox, C. S. (2009). Cellular ADMA: regulation and action. Pharmacological Research, 60, 448-460.

Teerlink, T., Nijveldt, R. J., de Jong, S., \& van Leeuwen, P. A. (2002). Determination of arginine, asymmetric dimethylarginine, and symmetric dimethylarginine in human plasma and other biological samples by high-performance liquid chromatography. Anal Biochem, 303, 131-137.

Thomas, D. D., Ridnour, L. A., Isenberg, J. S., Flores-Santana, W., Switzer, C. H., Donzelli, S., Hussain, P., Vecoli, C., Paolocci, N., Ambs, S., Colton, C. A., Harris, C. C., Roberts, D. D., \& Wink, D. A. (2008). The chemical biology of nitric oxide: implications in cellular signaling. Free Radical Biology \& Medicine, 45, 18-31.

Thum, T., Fraccarollo, D., Schultheiss, M., Froese, S., Galuppo, P., Widder, J. D., Tsikas, D., Ertl, G., \& Bauersachs, J. (2007). Endothelial nitric oxide synthase uncoupling impairs endothelial progenitor cell mobilization and function in diabetes. Diabetes, 56, 666-674.

Toyokuni, S. (2008). Molecular mechanisms of oxidative stress-induced carcinogenesis: from epidemiology to oxygenomics. IUBMB Life, 60, 441-447.

Turan, B., Tuncay, E., \& Vassort, G. (2012). Resveratrol and diabetic cardiac function: focus on recent in vitro and in vivo studies. J Bioenerg Biomembr, 44, 281-296.

Ueda, S., Kato, S., Matsuoka, H., Kimoto, M., Okuda, S., Morimatsu, M., \& Imaizumi, T. (2003). Regulation of cytokine-induced nitric oxide synthesis by asymmetric dimethylarginine: role of dimethylarginine dimethylaminohydrolase. Circulation Research, 92, 226-233.

Uriu-Adams, J. Y., \& Keen, C. L. (2005). Copper, oxidative stress, and human health. Mol Aspects Med, 26, 268-298.

Ushio-Fukai, M., Zafari, A. M., Fukui, T., Ishizaka, N., \& Griendling, K. K. (1996). p22phox is a critical component of the superoxide-generating NADH/NADPH oxidase system and regulates angiotensin II-induced hypertrophy in vascular smooth muscle cells. Journal of Biological Chemistry, 271, 23317-23321.

Valkonen, V. P., Paiva, H., Salonen, J. T., Lakka, T. A., Lehtimaki, T., Laakso, J., \& Laaksonen, R. (2001). Risk of acute coronary events and serum concentration of asymmetrical dimethylarginine. Lancet, 358, 2127-2128.

Vallance, P., \& Leiper, J. (2004). Cardiovascular biology of the asymmetric dimethylarginine:dimethylarginine dimethylaminohydrolase pathway. Arterioscler Thromb Vasc Biol, 24, 1023-1030.

Vallance, P., Leone, A., Calver, A., Collier, J., \& Moncada, S. (1992). Accumulation of an endogenous inhibitor of nitric oxide synthesis in chronic renal failure. Lancet, 339, 572-575. 
Vergely, C., Maupoil, V., Benderitter, M., \& Rochette, L. (1998). Influence of the severity of myocardial ischemia on the intensity of ascorbyl free radical release and on post-ischemic recovery during reperfusion. Free Radical Biology \& Medicine, 24, 470-479.

Vergely, C., Maupoil, V., Clermont, G., Bril, A., \& Rochette, L. (2003). Identification and quantification of free radicals during myocardial ischemia and reperfusion using electron paramagnetic resonance spectroscopy. Archives of Biochemistry \& Biophysics, 420, 209-216.

Vergely, C., Perrin-Sarrado, C., Clermont, G., \& Rochette, L. (2002). Postischemic recovery and oxidative stress are independent of nitric-oxide synthases modulation in isolated rat heart. $J$ Pharmacol Exp Ther, 303, 149-157.

Vergely, C., Tabard, A., Maupoil, V., \& Rochette, L. (2001). Isolated perfused rat hearts release secondary free radicals during ischemia reperfusion injury. Cardiovascular effect of the spin trap alpha-phenyl $N$-tert-butylnitrone. Free Radical Research, 35, 475-489.

Virdis, A., Neves, M. F., Amiri, F., Viel, E., Touyz, R. M., \& Schiffrin, E. L. (2002). Spironolactone improves angiotensin-induced vascular changes and oxidative stress. Hypertension, 40, 504510.

Vitecek, J., Lojek, A., Valacchi, G., \& Kubala, L. (2012). Arginine-based inhibitors of nitric oxide synthase: therapeutic potential and challenges. Mediators Inflamm, 2012, 318087.

Wadham, C., \& Mangoni, A. A. (2009). Dimethylarginine dimethylaminohydrolase regulation: a novel therapeutic target in cardiovascular disease. Expert Opin Drug Metab Toxicol, 5, 303-319.

Wang, D., Strandgaard, S., Iversen, J., \& Wilcox, C. S. (2009). Asymmetric dimethylarginine, oxidative stress, and vascular nitric oxide synthase in essential hypertension. Am J Physiol Regul Integr Comp Physiol, 296, R195-200.

Wang, J., Chen, L., Sinha, S. H., Liang, Z., Chai, H., Muniyan, S., Chou, Y. W., Yang, C., Yan, L., Feng, Y., Li, K. K., Lin, M. F., Jiang, H., Zheng, Y. G., \& Luo, C. (2012). Pharmacophore-based virtual screening and biological evaluation of small molecule inhibitors for protein arginine methylation. J Med Chem, 55, 7978-7987.

Werner, E. R., Blau, N., \& Thony, B. (2011). Tetrahydrobiopterin: biochemistry and pathophysiology. Biochemical Journal, 438, 397-414.

Wilcox, C. S. (2012). Asymmetric dimethylarginine and reactive oxygen species: unwelcome twin visitors to the cardiovascular and kidney disease tables. Hypertension, 59, 375-381.

Williams, G., Shi-Wen, X., Abraham, D., Selvakumar, S., Baker, D. M., \& Tsui, J. C. (2012). Nitric oxide manipulation: a therapeutic target for peripheral arterial disease? Cardiol Res Pract, 2012, 656247.

Wohlfart, P., Xu, H., Endlich, A., Habermeier, A., Closs, E. I., Hubschle, T., Mang, C., Strobel, H., Suzuki, T., Kleinert, H., Forstermann, U., Ruetten, H., \& Li, H. (2008). Antiatherosclerotic effects of small-molecular-weight compounds enhancing endothelial nitric-oxide synthase (eNOS) expression and preventing eNOS uncoupling. J Pharmacol Exp Ther, 325, 370-379.

Wu, G., \& Morris, S. M., Jr. (1998). Arginine metabolism: nitric oxide and beyond. Biochemical Journal, 336 ( Pt 1), 1-17.

Wunderlich, C., Schober, K., Schmeisser, A., Heerwagen, C., Tausche, A. K., Steinbronn, N., Brandt, A., Kasper, M., Schwencke, C., Braun-Dullaeus, R. C., \& Strasser, R. H. (2008). The adverse cardiopulmonary phenotype of caveolin-1 deficient mice is mediated by a dysfunctional endothelium. Journal of Molecular \& Cellular Cardiology, 44, 938-947.

Xia, N., Daiber, A., Habermeier, A., Closs, E. I., Thum, T., Spanier, G., Lu, Q., Oelze, M., Torzewski, M., Lackner, K. J., Munzel, T., Forstermann, U., \& Li, H. (2010). Resveratrol reverses endothelial nitric-oxide synthase uncoupling in apolipoprotein E knockout mice. J Pharmacol Exp Ther, 335, 149-154.

Xu, J., Wu, Y., Song, P., Zhang, M., Wang, S., \& Zou, M. H. (2007). Proteasome-dependent degradation of guanosine 5 '-triphosphate cyclohydrolase I causes tetrahydrobiopterin deficiency in diabetes mellitus. Circulation, 116, 944-953. 
Xue, F., Fang, J., Delker, S. L., Li, H., Martasek, P., Roman, L. J., Poulos, T. L., \& Silverman, R. B. (2011). Symmetric double-headed aminopyridines, a novel strategy for potent and membranepermeable inhibitors of neuronal nitric oxide synthase. J Med Chem, 54, 2039-2048.

Yan, J., Tie, G., \& Messina, L. M. (2012). Tetrahydrobiopterin, L-arginine and vitamin C actsynergistically to decrease oxidative stress, increase nitricoxide and improve blood flow after induction of hindlimbischemia in the rat. Mol Med, 18, 676-684.

Yang, Q., Xue, H. M., Wong, W. T., Tian, X. Y., Huang, Y., Tsui, S. K., Ng, P. K., Wohlfart, P., Li, H., Xia, N., Tobias, S., Underwood, M. J., \& He, G. W. (2011). AVE3085, an enhancer of endothelial nitric oxide synthase, restores endothelial function and reduces blood pressure in spontaneously hypertensive rats. British Journal of Pharmacology, 163, 1078-1085.

Yoshimatsu, M., Toyokawa, G., Hayami, S., Unoki, M., Tsunoda, T., Field, H. I., Kelly, J. D., Neal, D. E., Maehara, Y., Ponder, B. A., Nakamura, Y., \& Hamamoto, R. (2011). Dysregulation of PRMT1 and PRMT6, Type I arginine methyltransferases, is involved in various types of human cancers. Int J Cancer, 128, 562-573.

Yost, J. M., Korboukh, I., Liu, F., Gao, C., \& Jin, J. (2011). Targets in epigenetics: inhibiting the methyl writers of the histone code. Curr Chem Genomics, 5, 72-84.

Yu, J., Weiwer, M., Linhardt, R. J., \& Dordick, J. S. (2008). The role of the methoxyphenol apocynin, a vascular NADPH oxidase inhibitor, as a chemopreventative agent in the potential treatment of cardiovascular diseases. Curr Vasc Pharmacol, 6, 204-217.

Zairis, M. N., Patsourakos, N. G., Tsiaousis, G. Z., Theodossis Georgilas, A., Melidonis, A., Makrygiannis, S. S., Velissaris, D., Batika, P. C., Argyrakis, K. S., Tzerefos, S. P., Prekates, A. A., \& Foussas, S. G. (2012). Plasma asymmetric dimethylarginine and mortality in patients with acute decompensation of chronic heart failure. Heart, 98, 860-864.

Zeller, M., Korandji, C., Guilland, J. C., Sicard, P., Vergely, C., Lorgis, L., Beer, J. C., Duvillard, L., Lagrost, A. C., Moreau, D., Gambert, P., Cottin, Y., \& Rochette, L. (2008). Impact of asymmetric dimethylarginine on mortality after acute myocardial infarction. Arterioscler Thromb Vasc Biol, 28, 954-960.

Zhang, L., Rao, F., Zhang, K., Khandrika, S., Das, M., Vaingankar, S. M., Bao, X., Rana, B. K., Smith, D. W., Wessel, J., Salem, R. M., Rodriguez-Flores, J. L., Mahata, S. K., Schork, N. J., Ziegler, M. G., \& O'Connor, D. T. (2007). Discovery of common human genetic variants of GTP cyclohydrolase $1(\mathrm{GCH} 1)$ governing nitric oxide, autonomic activity, and cardiovascular risk. Journal of Clinical Investigation, 117, 2658-2671.

Zhang, Y. H., \& Casadei, B. (2012). Sub-cellular targeting of constitutive NOS in health and disease. Journal of Molecular \& Cellular Cardiology, 52, 341-350. 


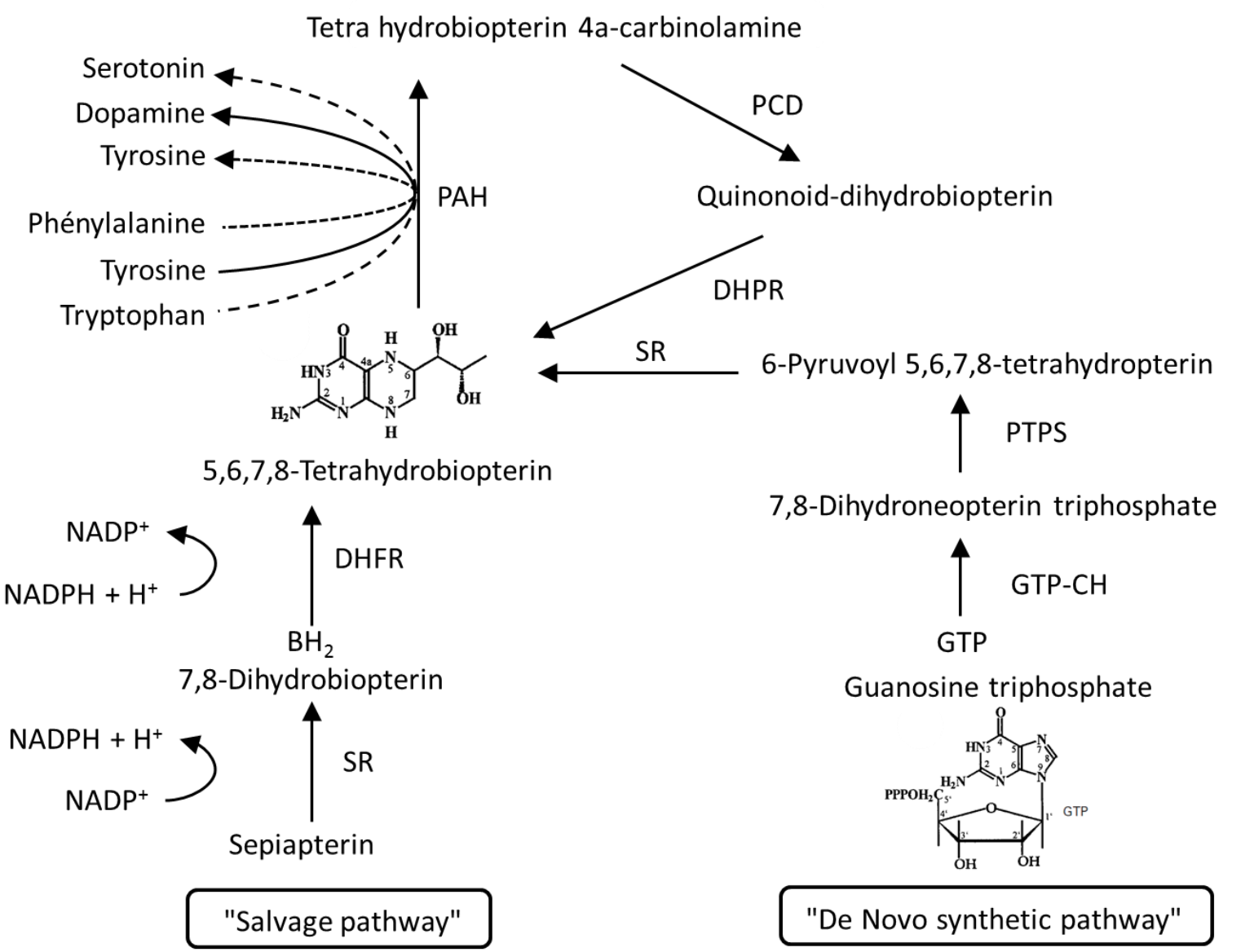

Figure 1: Pathways of tetrahydrobiopterin $\left(\mathrm{BH}_{4}\right)$ synthesis and degradation.

1) "De Novo synthetic pathway": $\mathrm{BH}_{4}$ biosynthesis proceeds from guanosine triphosphate (GTP) via 7,8-dyhydroneopterin triphosphate and 6-pyruvoyl-5,6,7,8-tetrahydropterin. The first and rate-limiting step in the pathway is GTP cyclohydrolase (GTP-CH). Subsequent steps are catalyzed by 1) 6-pyruvoyl tetrahydropterin synthase (PTPS) and sepiapterin reductase (SR).

2) A "salvage pathway" for $\mathrm{BH}_{4}$ synthesis: $\mathrm{BH}_{4}$ can be formed from exogenous sepiapterin. Sepiapterin is reduced by sepiapterin reductase (SR) to 7,8-dihydrobiopterin $\left(\mathrm{BH}_{2}\right)$ and further by dihydrofolate reductase (DHFR) to form $\mathrm{BH}_{4}$.

Regeneration of $\mathrm{BH}_{4}: \mathrm{BH}_{4}$ provides electrons for the hydroxylation of phenylalanine, tyrosine, and tryptophan by the action of phenylalanine hydroxylase (PAH), tyrosine hydroxylase (TH), and neuronal tryptophan hydroxylase (NTPH), respectively. During the catalytic event of aromatic amino acid hydroxylation, molecular oxygen is transferred to the corresponding amino acid and $\mathrm{BH}_{4}$ is oxidized to 4a-hydroxy-tetrahydrobiopterin. Two enzymes are involved in its subsequent reactions: PCD (pterin-4a-carbinolamine dehydratase) and DHPR (dihydropteridine reductase). 


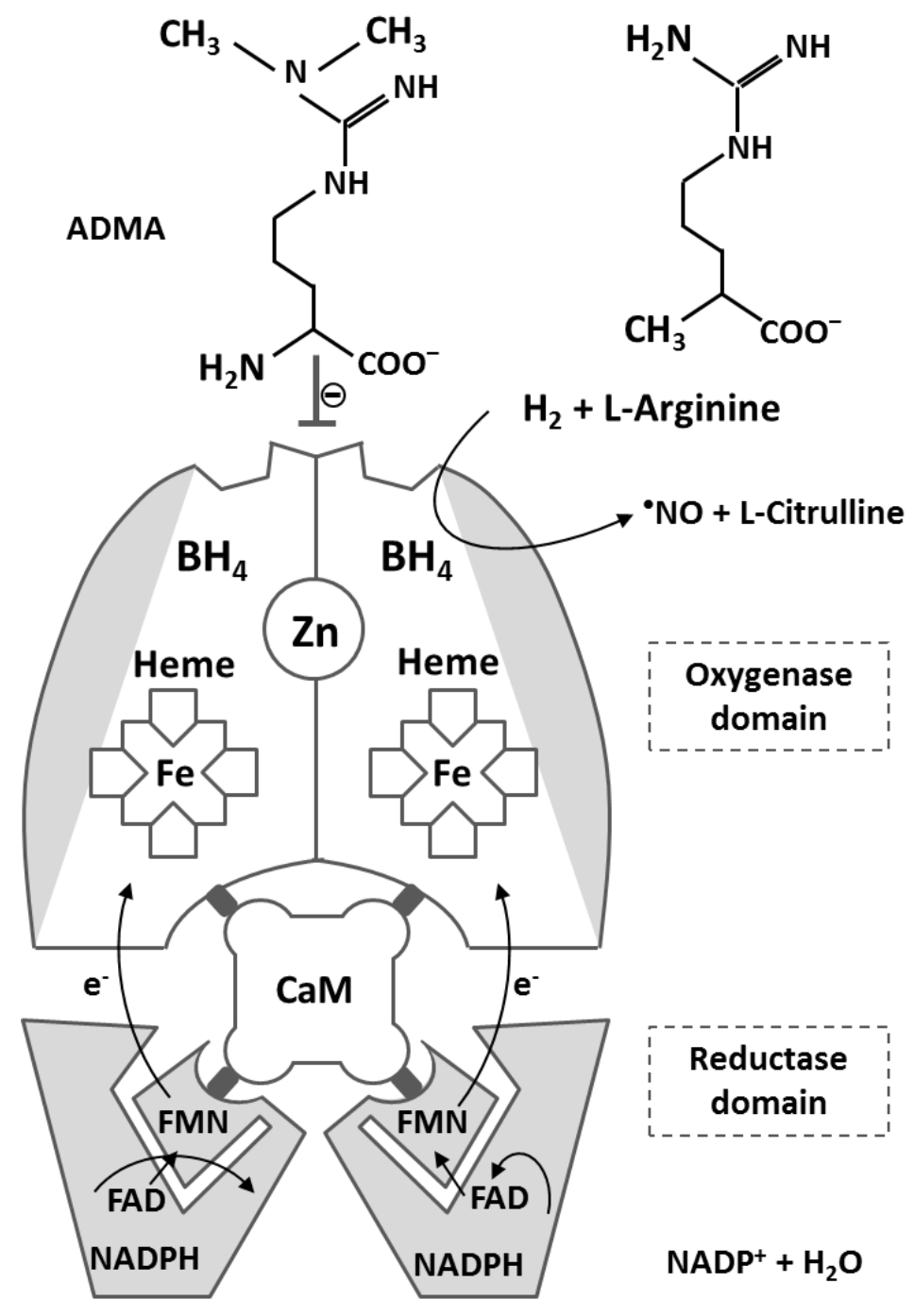

Figure 2: Molecular structures of the nitric oxide synthase (NOS) dimer.

The $\mathrm{N}$-terminal catalytic domain binds the heme prosthetic group as well as the redox cofactor, tetrahydrobiopterin $\left(\mathrm{BH}_{4}\right)$ associated with a regulatory protein, calmodulin (CaM). $\mathrm{BH}_{4}$ stabilizes the dimer. The $\mathrm{C}$-terminal reductase domain has the binding sites for flavin mononucleotide (FMN), flavin adenine dinucleotide (FAD), and nicotinamide adenine dinucleotide phosphate (NADPH). NOS produce nitric oxide (NO) from L-arginine (L-Arg) in the presence of $\mathrm{O}_{2}$ and NADPH. Zinc ( $\left.\mathrm{Zn}\right)$ is responsible for connecting the monomers to the heme groups. NADPH-oxidase donates an electron, which is ultimately used to convert L-Arg and $\mathrm{O}_{2}$ to the reaction products L-citrulline and NO. Asymmetric dimethylarginine (ADMA) inhibits NO synthesis by competing with L-Arg at the active site of NOS. 


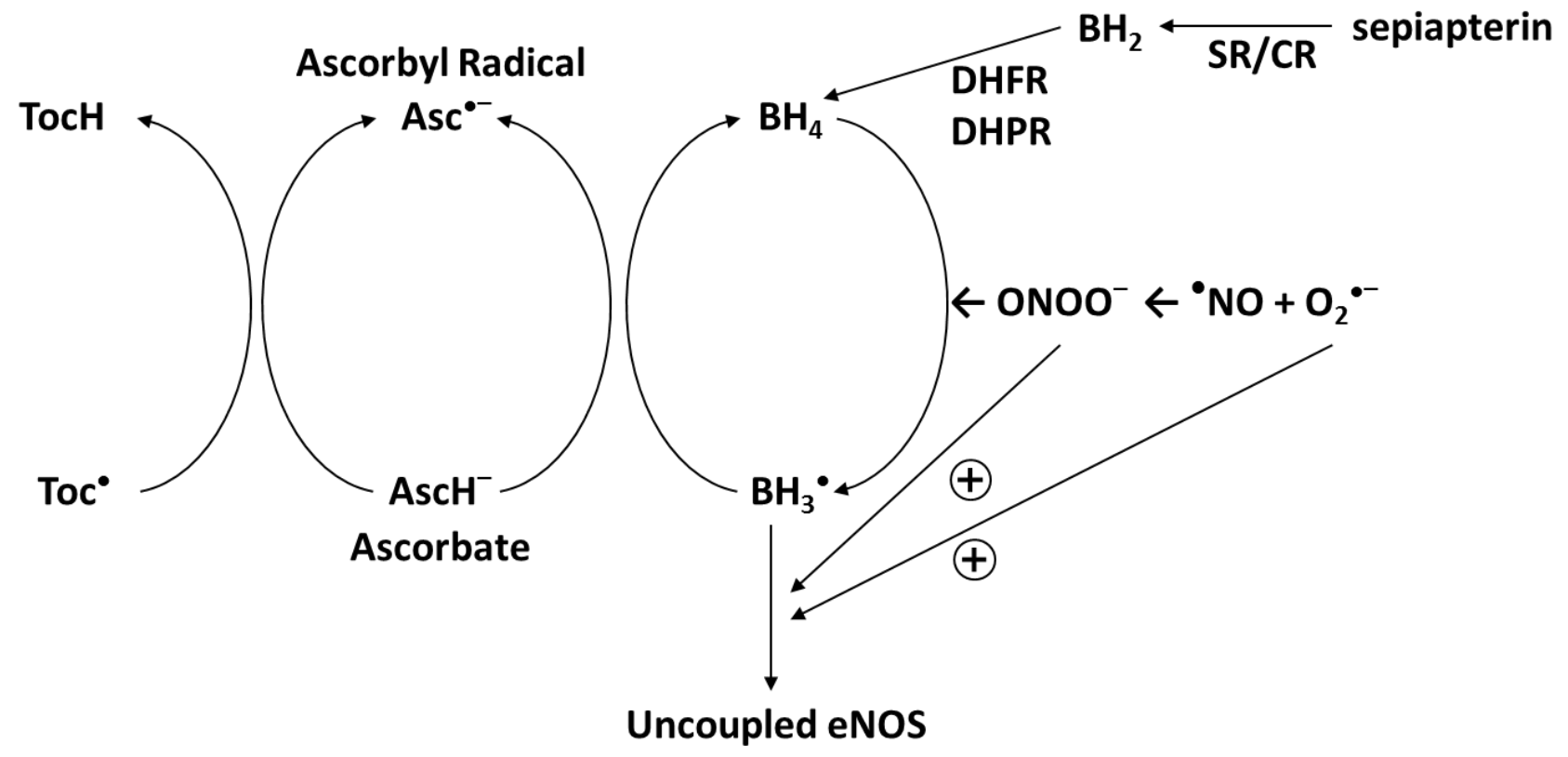

Figure 3. Reactions between $\mathrm{BH}_{4}$ and oxidative stress

Exogenous sepiapterin can be reduced by sepiapterin reductase (SR) or carbonyl reductase (CR) to dihydrobiopterin $\left(\mathrm{BH}_{2}\right)$, and further by dihydrofolate reductase (DHFR) and dihydropteridine reductase (DHPR) to form tetrahydrobiopterin $\left(\mathrm{BH}_{4}\right)$ (the so-called "salvage" pathway). Peroxynitrite (ONOO) oxidizes $\mathrm{BH}_{4}$ to the intermediate trihydrobiopterin radical $\left(\mathrm{BH}_{3}{ }^{\circ}\right)$ radical, which can be converted back to $\mathrm{BH}_{4}$ by ascorbate $\left(\mathrm{AscH}^{-}\right)$. Vitamin $\mathrm{E}$ (tocopherols, $\mathrm{TocH}$ ) is regenerated by the reaction of tocopheroxyl radical ( $\mathrm{Toc}^{\circ}$ ) with $\mathrm{AscH}^{-}$. Asc ${ }^{\circ}$ : Dehydroascorbate monoanion radical. 


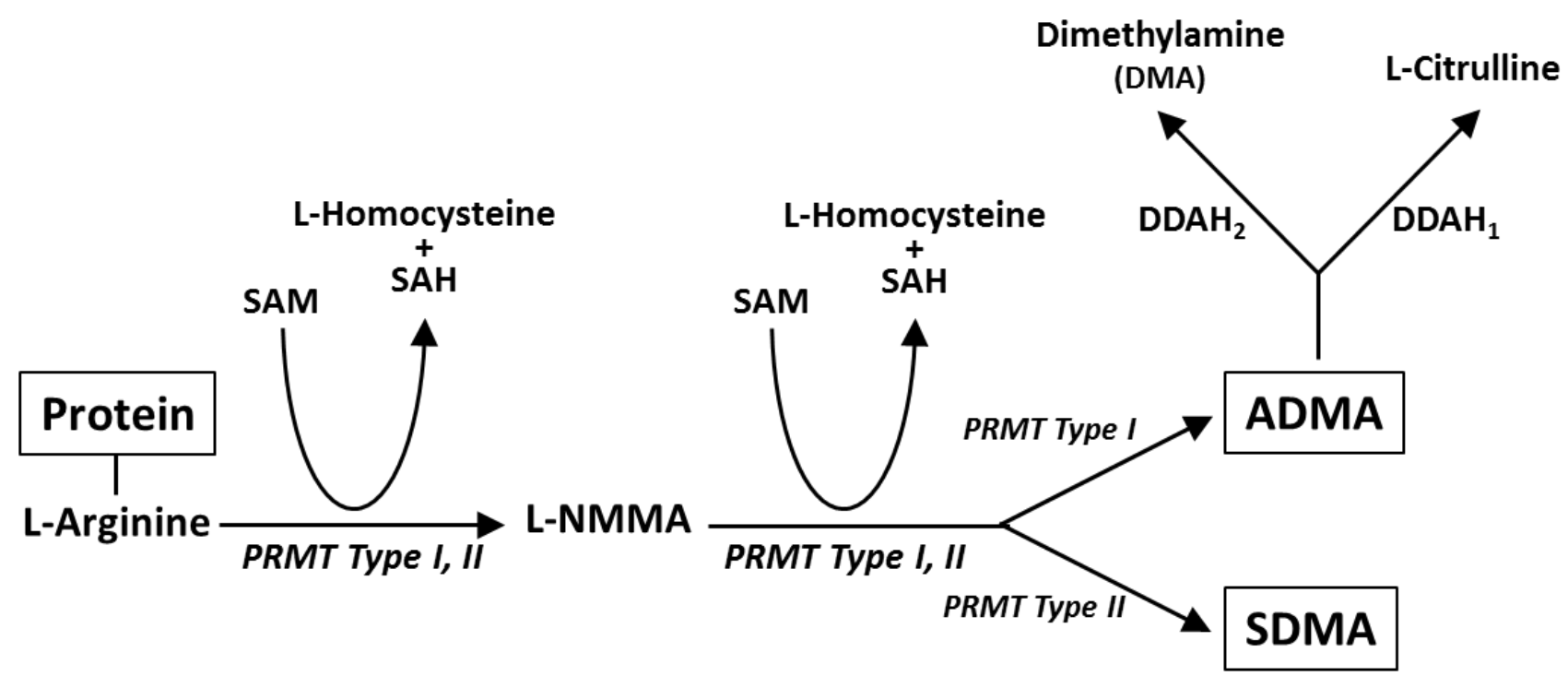

Figure 4: Metabolism of asymmetric dimethylarginine (ADMA)

ADMA is formed during the intracellular turnover of proteins. Methylated arginine residues are released from the breakdown of proteins that have been acted on by protein-arginine methyl transferases (PRMTs). These are classified into type I (PRMT1, 3, 4, 6, and 8) and type II (PRMT5, 7, and FBXO11) enzymes. PRMT type I and II catalyze the formation of $\mathrm{N}^{G}$-monomethyl-L-arginine (LNMMA) from L-arginine. In the second step, type I PRMTs produce ADMA, while type II PRMTs catalyze symmetric dimethylarginine (SDMA). ADMA can be degraded to citrulline and dimethylamines (DMA) by dimethylarginine dimethylaminohydrolases (DDAHs). SAM: S-adenosyl-Lmethionine; SAH: S-adenosyl-L-homocysteine 


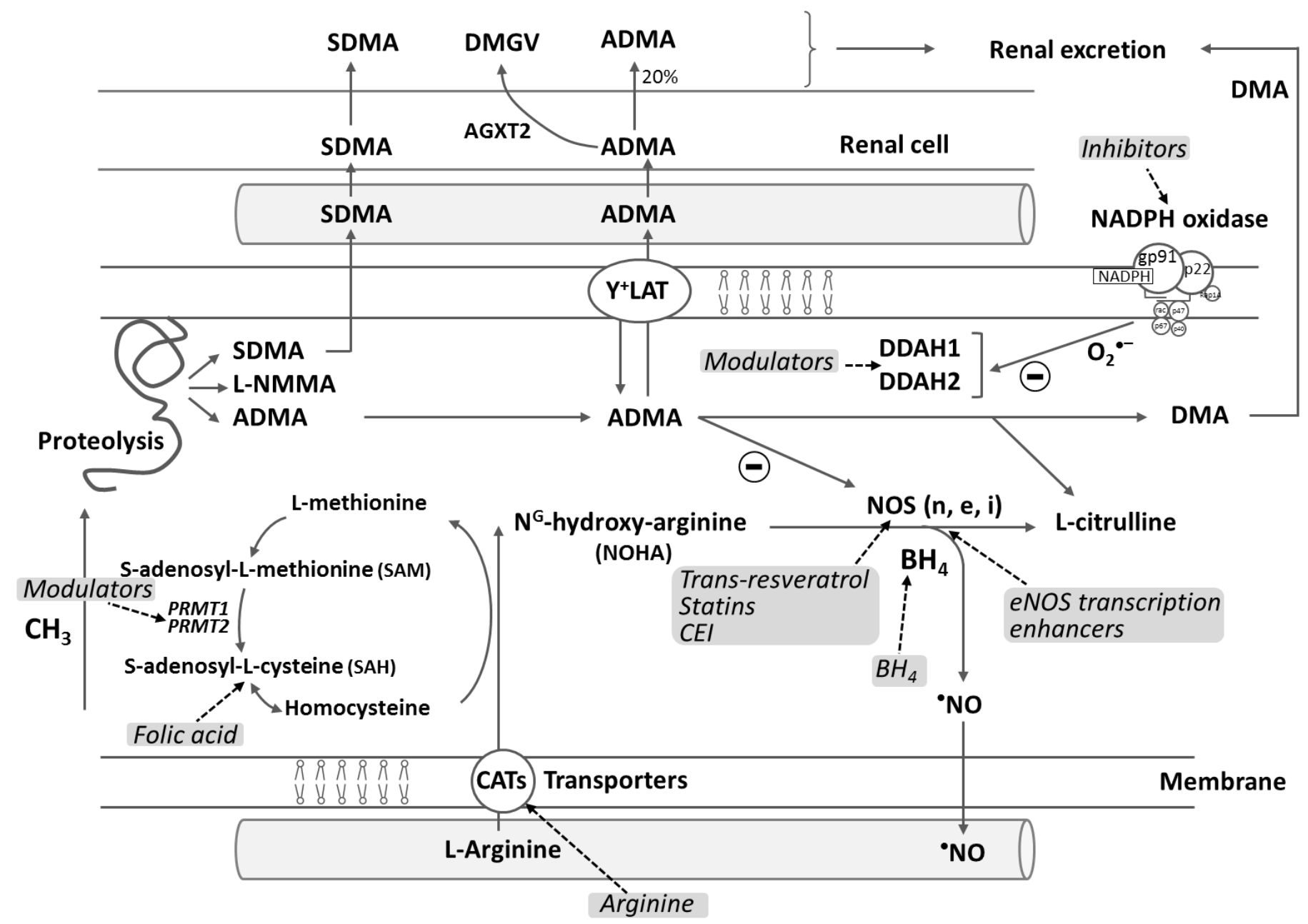

Figure 5. Pharmacological options to modulate the NO generating system in the vasculature.

This schema presents the sources and consequences of the production of nitric oxide (NO), asymmetric dimethylarginine (ADMA) and reactive oxygen species in intracellular and extracellular compartments. (see the details in the text). Pharmacological options (shaded parts): (1) Substrate availability: L-Arginine (2) eNOS transcription enhancers (3) treatment with $\mathrm{BH}_{4}$ (4) with folic acid (5) with trans-resveratrol (6) statins (7) drugs that interfere with the renin-angiotensin-aldosterone system (8) modulation of DDAH and PRMT activities and (9) selective NADPH oxidase inhibitors. 
A. $\begin{cases}1 . & \text { L-Arginine } \\ 2 . & \text { L-NMA }\end{cases}$<smiles>N=C(N)NCCCC(N)C(=O)O</smiles>

3. Dipeptide agent

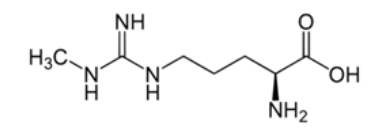

4. Double-headed symmetric inhibitor

5. Cis-3,4-pyrrolidine-based inhibitor

B.

6. Aromatic 4-oxo-pteridine inhibitor

C.

7. Thioether-based inhibitor
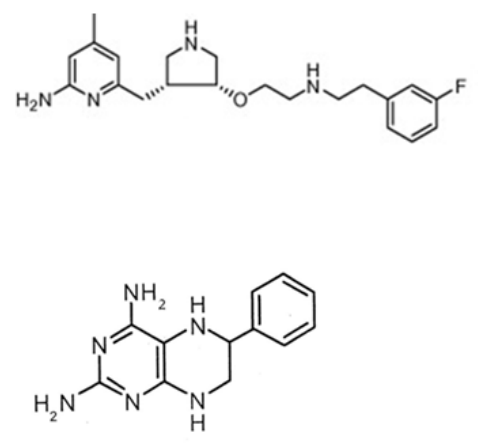

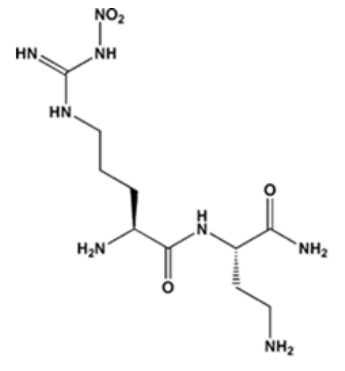

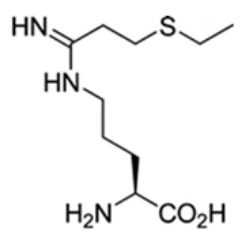

Figure 6. Chemical structure of typical nitric oxide synthase inhibitors
A. Substrate based inhibitors
B. Compounds interacting with tetrahydrobiopterin
C. Inhibitors interacting with the heme 